\title{
Evolution of Pre-Collective Nuclei: Structural Signatures Near the Drip Lines
}

R. F. Casten ${ }^{1}$ and N. V. Zamfir $1,2,3$

1. Brookhaven National Laboratory, Upton, NY, 11973, USA

2. Clark University, Worcester, MA, 01610, USA

3. Institute of Atomic Physics, Bucharest Magurele, Romania

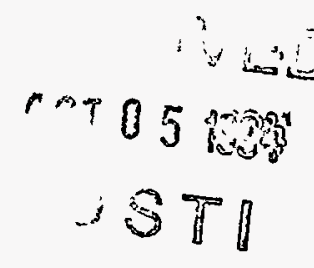

$3 \mathrm{STl}$

\begin{abstract}
$\underline{\text { Abstract }}$
Recent studies have shown that the phenomenology of single-magic and nearmagic nuclei has universal characteristics analogous to those of collective nuclei and that, moreover, this phenomenology attaches smoothly to that describing collective nuclei. This has led to a number of new signatures of structure as well as to a new, tripartite, classification of nuclear structure that embraces the gamut of structures from magic, through pre-collective, to fully collective and rotational nuclei. Aside from the natural appeal of simple global correlations of collective observables, these results have particular significance for soon-to-be accessible exotic nuclei near the drip lines since they rely on only the simplest-to-obtain data, in particular, the energies of just the first two excited states, $E\left(4_{1}^{+}\right)$and $E\left(2_{1}^{+}\right)$, of eveneven nuclei, and the $B\left(E 2: 2_{1}^{+} \rightarrow 0_{1}^{+}\right)$value. Indeed, without the need for more extensive level schemes, these basic data alone can reveal information about the goodness of seniority, about the validity of pair-addition mode relationships of adjacent even-even nuclei, about underlying shell structure (validity of magic numbers) and even about the shell model potential itself (e.g., the strengths of the $l \cdot s$ and $l^{2}$ terms).
\end{abstract}




\section{Introduction}

The advent of a variety of radioactive nuclear beam (RNB) facilities worldwide in the next decade will open up entirely new horizons for nuclear structure. It will be interesting to study new examples of the kinds of structure familiar from known nuclei. However, the real excitement of exotic nuclei far from stability centers on the possibility that radically new nuclear properties will be encountered, unlike any known to date. This is especially likely near the neutron drip line.

These speculations are based on a number of factors and are backed by important recent calculations. ${ }^{1}$ Consider the schematic single-particle levels in fig. 1. Near the neutron drip it is likely that the residual $p-n$ interaction will be quite different than it is nearer stability because the large neutron excess implies that protons and neutrons will be filling rather different orbits. Since it is this interaction which is primarily responsible for configuration mixing, deformation and the development of collectivity in nuclei, it is reasonable to expect that such facets of structure might also be quite different.

Likewise, the pairing interaction--which is the main competitor to the $p-n$ interaction in determining structure--will almost certainly have quite different manifestations. The pairing interaction normally scatters pairs of particles amongst the single-particle levels near the Fermi surface. When some of these levels are unbound, as occurs near the drip line, significant changes are expected.

Occupation of unbound or near-unbound levels could lead to a type of neutron skin or low density neutron gas surrounding a less neutron-rich core. There have already been speculations of possible core-skin collective modes such as mini-giant resonance oscillations of one against the other. ${ }^{2}$ There are analogies (but also differences) between this skin region and the neutron halos that have recently been so extensively studied in light neutron-rich nuclei. 


\section{DISCLAIMER}

This report was prepared as an account of work sponsored by an agency of the United States Government. Neither the United States Government nor any agency thereof, nor any of their employees, make any warranty, express or implied, or assumes any legal liability or responsibility for the accuracy, completeness, or usefulness of any information, apparatus, product, or process disciosed, or represents that its use would not infringe privately owned rights. Reference herein to any specific commercial product, process, or service by trade name, trademark, manufacturer, or otherwise does not necessarily constitute or imply its endorsement, recommendation, or favoring by the United States Government or any agency thereof. The views and opinions of authors expressed herein do not necessarily state or reflect those of the United States Government or any agency thereof. 


\section{DISCLAIMER}

Portions of this document may be illegible in electronic image products. Images are produced from the best available original document. 
Near the drip line the quantum mechanics necessarily will involve the interaction of bound and resonance or continuum states, and this should lead to single-particle and collective phenomena qualitatively different than those near stability. Finally, as single-particle levels merge into the continuum, the traditional symmetries of shell structure--SU(3) in light nuclei, pseudo-SU(3) in heavy nuclei-may lose relevance. There are two reasons for this. First, the sequencing and spacing of orbits will be different and, secondly, some orbits will be lost to the continuum, leading to the loss of a key characteristic of the shell structure of normal parity orbits near stability, namely the presence of all $j$ values from $1 / 2$ to some maximum value.

Some of these ideas have taken concrete form in some important recent calculations ${ }^{1,3}$ which have presented some enticing results of relativistic mean-field calculations near the neutron drip line. These calculations suggest, for example, that even the shell-model central potential itself may not be the same. Specifically, they suggest that the $l^{2}$ term may be absent or significantly smaller than near stability. While this specific prediction may or may not be substantiated, it indicates the kind of effects that might come into play. The consequences of such effects are hard to know in detail at this time--ref. 3 itself warns of the dangers of using conventional calculational approaches in unconventional territory. But, it is equally hard to overestimate the importance of refs. 1,3. For example, the absence of an $l^{2}$ term, even viewed in a conventional shell model framework, will certainly alter the magic numbers. More than this, it will alter the familiar sequencing of shell model orbits that proceeds more or less smoothly from high $j$ values early in a shell to low $\mathrm{j}$ at the top. Rather, the low $\mathrm{j}$ orbits will be concentrated near mid-shell. In addition, in medium and heavy mass nuclei, there would be no unique parity orbit and the myriad effects of this orbit at both low and high spin would therefore vanish. 
These provocative efforts 1,3 to probe the structure of medium and heavy drip line nuclei have set the stage for high expectations of exotic phenomena. At the same time, the results they embody will hardly be the last word. Other calculations ${ }^{4}$ already have reached somewhat different conclusions. The key point is that all the novel ingredients are at hand near the neutron drip line to foster new structural phenomena. Hopefully, these early forays into unknown territory will engender a greatly expanded theoretical effort to provide realistic predictions for the new nuclear regions that will soon be accessible, both to test our ideas of structure and to serve as preliminary guidelines for the choices of key experiments.

It is the purpose of this paper to take off from these kinds of speculations and to look at some of the possible experimental signatures of radically new structure. While the present results will be based as much as possible on the behavior of known nuclei and on the predictions of standard models, everything said above urges caution in blindly accepting the conclusions. Rather, we hope that the present results may encourage better and more sophisticated studies of the empirical consequences of the type of study Nazarewicz and colleagues have initiated. We will illustrate some of these ideas with predictions based on the specific suggestion of ref. 1, but the qualitative results we discuss are not wedded to any specific prediction. The focus will be on singly magic nuclei or those near closed shells, but occasional comments on collective and deformed nuclei will be added.

II. Near-Magic Nuclei

\section{A. Basic Concepts}

The standard approach to nuclei near closed shells is the shell model with short range and attractive residual interactions. Due to the relative weakness of the nuclear force, deeply bound nucleons are generally not excited to open shells in nucleon collisions/interactions. Therefore, the principal determinants of structure are the valence nucleons and the interactions amongst them. The residual valence 
$\mathrm{T}=0 \mathrm{p}-\mathrm{n}$ interaction is the most important factor in the onset and development of collectivity and deformation but plays a lesser role near closed shells and, to first order, none at all in magic nuclei. For simplicity in most of what follows, we will deal with singly magic nuclei, and hence we will focus on the like nucleon interaction. In $\mathrm{J}=0^{+}$states this exhibits the pairing property to good approximation, but other aspects are extremely important for $\mathrm{J} \neq 0^{+}$states.

To start, we summarize two key properties of like-nucleon systems interacting with short-range attractive forces, namely the spectrum of a 2-particle configuration in a single j-orbit, and the consequences of the seniority scheme. We approximate the residual interaction with a surface $\delta$ force. Results with realistic interactions will differ in detail, but the key physics is almost always discernable with a $\delta$ interaction-at least in nuclei familiar to us; whether this persists near the drip lines is an interesting question. Consider the configuration of a pair of nucleons in an orbit $j$, $\left|j^{2} J\right\rangle$, where $J=0,2, \ldots . .2 j-1$. Regardless of the value of $j$, the same type of level spectrum results. The $0^{+}$state in which the orbits of the two particles are co-planar lies lowest, by far, due to the strong attractive interaction when the two particles are, on average, closest to each other. The angles between the angular momentum vectors of the particles in the higher $j$ states increase, hence their average internucleon distance increases, and the effect of the $\delta$ interaction decreases. [The interaction is weakest for the $J_{\max }$ state even though the orbits are again nearly coplanar because of the effects of total anti-symmetrization arising from the Pauli Principle.] The resultant $\left|\mathrm{j}^{2} \mathrm{~J}\right\rangle$ spectrum is as shown in fig. 2. It is nearly independent of $j$. The key quantity

$$
\mathrm{R}_{4 / 2} \equiv \mathrm{E}\left(4_{1}^{+}\right) / \mathrm{E}\left(2_{1}^{+}\right)
$$

is in the range 1.15-1.20 for all $j$ values encountered. 
Two extensions of these ideas to more than two particles and more than one $j$ value allow the treatment of many realistic nuclei near closed shells. The first extension centers around the seniority concept, the second around configuration mixing. [Note that the latter is tantamount to, and essential for, deformation since it inherently involves an uneven distribution of $m$-substates.] The seniority scheme and its ramifications are fundamental to much of nuclear structure. Its essentials can even be discerned in the highly broken seniority environment of deformed nuclei. Basically, it provides a simple method to deduce, predict, and understand the properties of $n$-body systems from those of fewer-body systems (in practice, usually in terms of 2-body systems).

There are two particular results of the seniority scheme that are critical to our discussion. It is simple to show 5,6 , in a $\mathrm{j}^{\mathrm{n}}$ configuration with good seniority, $\mathrm{v}$, that the excitation energies of all states are constant, independent of $n$. That is, the excitation energies of states $\left|j_{V}^{n} J\right\rangle$ are identical to those of $\left|j_{V}^{v} J\right\rangle$. In particular, for seniority $\mathrm{v}=2$

$$
E\left(j_{v=2}^{n} J\right)=E\left(j_{v=2}^{2} J\right)
$$

This is illustrated in fig. 3. The classic empirical example of this, made justly famous by Talmi, is the $\mathrm{Sn}$ isotopes, shown in fig. 4 .

Another key observable that acts as a signature of structure is the $B(E 2)$ value to the first $2^{+}$state, $\mathrm{B}\left(\mathrm{E} 2: 2_{1}^{+} \rightarrow 0_{1}^{+}\right)$. [Since we will nearly always focus on this particular $B(E 2)$ below, we will hereafter usually abbreviate it to just $B(E 2)$.] The seniority scheme predicts a very specific $n$-dependence of $B(E 2)$ values in $j^{n}$ configurations. Taking the $2_{1}^{+}$state as having $v=2$, we have

$$
\mathrm{B}\left(\mathrm{E} 2: 2_{1}^{+} \rightarrow 0_{1}^{+}\right)_{\mathrm{n}}=\frac{\mathrm{n}(2 \mathrm{j}+1-\mathrm{n})}{2(2 \mathrm{j}-1)} \mathrm{B}\left(\mathrm{E} 2: 2_{1}^{+} \rightarrow 0_{1}^{+}\right)_{2}
$$


Therefore, a good approximation to the n-dependence of the $B(E 2)$ values is 6

$$
\mathrm{B}\left(\mathrm{E} 2: 2_{1}^{+} \rightarrow 0_{1}^{+}\right)_{\mathrm{n}} \propto \mathrm{f}(1-\mathrm{f})
$$

where $f=\frac{n}{2 j+1}$ is the fractional occupation of the shell. This bell-shaped dependence peaking at mid-shell is illustrated in fig. 5. The qualitatively different behavior of energies and $B(E 2)$ values ultimately originates in the properties of odd and even tensor operators in 2-particle systems.

\section{B. Comparison with Data: Global Phenomenology}

The comparison of eqs. 2 and 4 with the data is interesting and provides clues to what may happen far from stability. Although the constancy of $2_{1}^{+}$and $4_{1}^{+}$energies in $\mathrm{Sn}$ is well known, it is not the general case. This is illustrated in figs. $6 a, b$ which shows $\mathrm{E}\left(2_{1}^{+}\right), \mathrm{E}\left(4_{1}^{+}\right)$and their energy difference

$$
\Delta \mathrm{E}_{4-2} \equiv \mathrm{E}\left(4_{1}^{+}\right)-\mathrm{E}\left(2_{1}^{+}\right)
$$

for singly magic nuclei. These data reveal an interesting behavior. While, in general, neither $\mathrm{E}\left(2_{1}^{+}\right)$nor $\mathrm{E}\left(4_{1}^{+}\right)$is constant, they vary together so that their energy difference $\Delta \mathrm{E}_{4-2}$ is nearly constant. An alternate form of eq. 5 with constant $\Delta \mathrm{E}_{4-2}$ is

$$
\mathrm{E}\left(4_{1}^{+}\right)=\mathrm{E}\left(2_{1}^{+}\right)+\text {constant }
$$

That is, $\mathrm{E}\left(4_{1}^{+}\right)$should be linear against $\mathrm{E}\left(2_{1}^{+}\right)$with a slope of unity. This is in fact the case as fig. 7 shows for several mass regions. The data are consistent with slopes very close to unity, except in the actinides. This phenomenology is incompatible with the seniority scheme. Note that an explanation of this does not simply 
involve extension to a multi-j environment. For example, several degenerate $j$ orbits interacting with an odd tensor interaction (e.g., a $\delta$ force) will have good generalized seniority in which the usual seniority predictions are retained.

To attempt to understand the empirical results of fig. $6 \mathrm{a}, \mathrm{b}$ we have carried out simple shell model calculations with a surface $\delta$ interaction. The results ${ }^{7}$ for some of the neutron magic numbers are shown in fig. $6 \mathrm{c}$ and at the bottom of fig. 7. The calculations employed a variety of single particle energies, interaction strengths and orbits. Yet all lead to the same result-a slope of $\sim 1.0$ in eq. 6 corresponding to nearly constant $\Delta \mathrm{E}_{4-2}$. The same result emerges from realistic shell model calculations in medium and heavy nuclei. 8

Before we interpret these results, we consider the $\mathrm{B}(\mathrm{E} 2)$ values. Figure $8 \mathrm{a}$ shows $B(E 2)$ values against $A$ for singly magic nuclei. No obvious pattern emerges. However, fig. $8 \mathrm{~b}$ shows the same data plotted against the fractional shell filling $n /(2 j+1)$ where in this case $2 j+1$ is taken as the full major shell degeneracy. Superposed on the data is the prediction of eq. 4. The agreement is excellent.

This pair of empirical results, figs. $6 \mathrm{a}, \mathrm{b}$ and fig. $8 \mathrm{~b}$, seem contradictory: the energies point to a breakdown of seniority, but the $B(E 2)$ values increase with shell filling in a manner expected in the seniority scheme.

It is possible to understand this seemingly contradictory situation by a schematic two-j orbit calculation that isolates the essential physics behind the

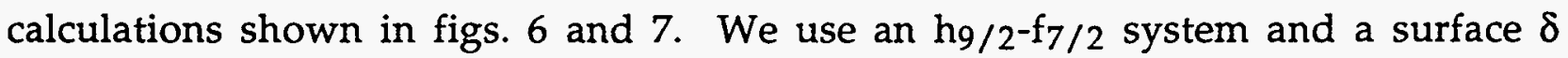
interaction. As in figs. 6 and 7 , the yrast energies vary, but $\Delta \mathrm{E}_{4-2}$ is quite constant. Table 1 shows the major components of the calculated wave functions (in the column labeled "exact"). Analysis of these wave functions shows that seniority and generalized seniority are indeed broken but that a simple pair addition behavior retains validity. In this picture a state of spin $\mathrm{J}$ in a singly magic nucleus with $\mathrm{n}$ valence nucleons is constructed by adding a pair of nucleons coupled to $0^{+}$to the 
state of the same spin in the nucleus with n-2 particles. Moreover, this 2-particle $0^{+}$ state has the same correlated wave function as the $0^{+}$ground state of the twovalence nucleon nucleus. Given the wave functions for different J values in the $n=2$ nucleus, those for all others are obtained merely by the successive addition of $0^{+}$-coupled pairs. These pair wave functions are easily constructed from the $n=2$ wave functions in the table and are given in the "pair" column. The agreement with the exact wave functions is excellent. The energies $\mathrm{E}\left(4_{1}^{+}\right)$and $\mathrm{E}\left(2_{1}^{+}\right)$can vary with $\mathrm{n}$ in such a scheme but their energy difference remains constant (i.e., they vary in energy together) since both are constructed by addition of the same $0^{+}$-coupled 2particle wave function. [Their excitation energies vary since the ground state binding energy is affected by the indistinguishability of its $0^{+}$-coupled pair constituents.]

In contrast to the energies, a calculation of the B(E2) values in the pair addition scheme does not lead to results qualitatively different from the seniority scheme of fig. $8 \mathrm{~b}$. The $B(E 2)$ values increase with $\mathrm{n}$ in the pair addition scheme as well. Indeed, it is difficult to concoct a model in which the $B(E 2)$ values do not increase with valence nucleon number. The model differences show up in different functional forms for that increase. For example, simple calculation with eq. 3 for $j=13 / 2$ gives $B(E 2)_{n=4}=1.67 B(E 2)_{n=2}$ while the pair addition result is a factor of 2 , which is almost the same.

Thus the energies of the $2_{1}^{+}$and $4_{1}^{+}$states, perhaps combined with $\mathrm{B}(\mathrm{E} 2)$ values, are a sensitive indicator of structure. Constant energies suggest a good seniority description. Varying energies with constant energy difference seem to point to a pair addition scheme of configuration-mixed states. A breakdown of this scheme should lead to $E\left(2_{1}^{+}\right), E\left(4_{1}^{+}\right)$, and $\Delta E_{4-2}$ values that vary with $n$.

For singly magic nuclei, it may be possible to glean some ideas of the number of valence nucleons of the active type from the $B(E 2)$ values by comparison with the 
fractional filling plot of fig. 8. Early on in a shell, the B(E2) values should increase roughly linearly with active valence nucleon number, while near mid-shell, they should be approximately flat.

In fig. 7 , although the slope of $\mathrm{E}\left(4_{1}^{+}\right)$against $\mathrm{E}\left(2_{1}^{+}\right)$is constant, the ratio $R_{4 / 2}$ is continually changing, as is clear by rewriting eq. 6 as

$$
\mathrm{R}_{4 / 2}=\mathrm{E}\left(4_{1}^{+}\right) / \mathrm{E}\left(2_{1}^{+}\right)=1.0+\frac{\text { constant }}{\mathrm{E}\left(2_{1}^{+}\right)}
$$

An approximate $R_{4 / 2}$ scale is shown along the top in fig. 7. As $E\left(2_{1}^{+}\right)$decreases in fig. $7, R_{4 / 2}$ approaches the regime of collective values of 2 or more. An interesting aspect of fig. 7 is related to these $R_{4 / 2}$ values. Even though the slopes for different mass regions (except the actinides) are the same (unity), and even though the intercepts are nearly the same $(\sim 600 \mathrm{keV}), \mathrm{R}_{4 / 2}$ values for nuclei in the different regions are not identical, especially for magic nuclei. This occurs because $R_{4 / 2}$ is correlated with $\mathrm{E}\left(2_{1}^{+}\right)$--compare top and bottom scales--and magic nuclei in different regions have $2_{1}^{+}$energies that differ. Thus it is interesting to separately study $R_{4 / 2}$ values. We will see that they have a deep connection to the underlying shell structure and can act as signatures of new types of shell structure.

To pursue this we first recall that 2-identical particles in a single-j shell have $R_{4 / 2} \sim 1.2$. Similar $R_{4 / 2}$ values result for multi-j configurations of the form $\left(\mathrm{j}_{1}^{\mathrm{n}_{1}} \mathrm{~J}_{1}, \mathrm{j}_{2} \mathrm{~J}_{2} \ldots . . \mathrm{j}_{\mathrm{k}} \mathrm{n}_{\mathrm{k}} \mathrm{J}_{\mathrm{k}} \mathrm{J}\right)$ with $\mathrm{n}_{\mathrm{i}}$ even. Thus it is hardly expected that some of the best "shell model" nuclei do not have low $R_{4 / 2}$ values. Indeed, some of these $R_{4 / 2}$ approach collective values. For example, for ${ }^{18} \mathrm{O}$ and ${ }^{42} \mathrm{Ca}, \mathrm{R}_{4 / 2} \sim 1.8$. In contrast $\mathrm{R}_{4 / 2}$ is indeed $\sim 1.2$ in $\mathrm{Sn}$, and for ${ }^{134} \mathrm{Te},{ }^{210} \mathrm{~Pb}$ and ${ }^{210} \mathrm{Po}$. Some of the $\mathrm{R}_{4 / 2}$ data for magic nuclei is plotted in fig. 9 as a function of A.

The results are intriguing and heretofore unrecognized ${ }^{9}$. There is an extremely systematic behavior, with $R_{4 / 2}$ steadily decreasing toward " $\mid j^{2} J>$--values in heavy 
nuclei. The effects at work here are simple and useful to understand. Consider fig. 10 which shows angular momentum vector diagrams for $2^{+}$states constructed from 2 particles, one each occupying orbits $j_{1}$ and $j_{2}$. The case on the right is typical of most cases in which the $2^{+}$level is constructed by coupling the two angular momenta at some angle between their orbital planes. The $4^{+}$state corresponds to a somewhat larger angle. With a short range (e.g., $\delta$ ) force between the particles, the energy difference in the interaction for these two angles is small. The orbital planes are already far apart enough in the $2^{+}$state that there is little interaction. Hence the spectrum here is similar to that shown in fig. 2.

The left side of fig. 10, though, shows a special case. Suppose $j_{1}=j_{2} \pm 2$. Then, for this case only, the $2^{+}$state is constructed with co-planar orbits. Just as pairwise co-planar occupation of the same orbit leads to a low-lying $0^{+}$state in the pairing case, here occupation of two different orbits, with $\Delta \mathrm{j}= \pm 2$, leads to a large attractive interaction in the $2^{+}$state. This state therefore drops in energy, and $R_{4} / 2$ consequently rises. This is shown by the simple $\delta$ function calculations at the bottom of the figure. Generalizing this, it turns out ${ }^{9}$ that $R_{4 / 2}$ is very closely correlated in multi-j shell-model calculations with the summed probability for the occupation of $\Delta j= \pm 2$ orbit pairs by odd numbers of nucleons. We call this probability $\Sigma a_{o d d-n, \Delta j=2}^{2}$ or $\Sigma a^{2}$, for short. The calculated $R_{4 / 2}-\Sigma a^{2}$ correlation is illustrated for the 50-82 shell in fig. 11.

It is now immediately clear why the apparently strange phenomenology of $R_{4 / 2}$ in fig. 9 occurs. In light nuclei, each shell (e.g., $8-20$ or $20-40$ ) has a low-lying $\Delta \mathbf{j}=2$ pair ( $d_{5 / 2}-s_{1 / 2}$ and $f_{7 / 2}-p_{3 / 2}$, respectively). [Note, incidentally, that pairs such as $d_{3 / 2^{-}}$ $s_{1 / 2}$ or $p_{3 / 2}-p_{1 / 2}$ are also $\Delta j=2$.] Hence, the $2^{+}$state is lowered and $R_{4 / 2}$ is large. The values of $\sim 1.7$ found experimentally are easy to reproduce in shell model calculations (see fig. 11). In medium and heavy nuclei, however, the $l \cdot s$ and $l^{2}$ terms in the shell model Hamiltonian force the unique parity orbit down into the next 
lower shell, leaving no low-lying $\Delta \mathrm{j}=2$ pair. For example, in the $82-126$ shell the lowest orbits are $h_{9 / 2}$ and $f_{7 / 2}$. In these cases, there is no anomalous lowering of $E\left(2_{1}^{+}\right)$, the wave function remains dominated by configurations with even- $n$ occupation of each $j$ orbit, and $R_{4 / 2}$ is close to shell model values for $\left|j^{2} J\right\rangle$ configurations. Simple calculations ${ }^{9}$, one with constant interaction strength, $\mathrm{V}_{0}$, and one with decreasing strength, are shown in fig. 12. The concordance with the trend in fig. 9 is immediately obvious. Finally, it is worth noting an interesting feature of this discussion of odd $n-\Delta \mathbf{j}=2$ configurations in pre-collective nuclei: such "particle-hole" type excitations link naturally onto the characteristic "RPA-wave functions" of collective nuclei. Pursuit of this idea may help understand the transition from pre-collective to collective structures at $R_{4 / 2} \sim 2.0$.

\section{New Closed Shell Regions:}

\section{A. New Signatures of Structure}

With RNB facilities it is likely that new closed shell or near-closed shell regions will be encountered. More generally, new nuclei with "pre-collective" structure $\left(R_{4 / 2}<2.0\right)$ are likely to be discovered. New magic numbers may be identified and traditional ones may lose their validity. The latter, in fact, has already occured in the $\mathrm{N}=20\left({ }^{32} \mathrm{Mg}\right)$ and $\mathrm{Z}=40\left({ }^{80} \mathrm{Zr}\right.$ and $\left.{ }^{100} \mathrm{Zr}\right)$ regions. Shell structure, even close to the valley of stability, is hardly immutable: witness the appearance and disappearance of the $Z=38$ or 40 and $Z=64$ (sub)-shell gaps or even the relative change in the $1 g 7 / 2$ orbit between $91 \mathrm{Zr}$ and ${ }^{131} \mathrm{Sn}$. The question arises then whether some of the above ideas and phenomenology may provide new signatures of perhaps exotic structures.

How would one identify potential magic nuclei in new regions, in particular, near the neutron drip line, where radical departures from traditional structure are anticipated? The traditional requirement that the first excited states are high lying is perhaps too vague, as an absolute standard of comparison may not be well established. $R_{4 / 2}$ alone is clearly not a good indicator of nuclei near magic numbers 
since $18 \mathrm{O},{ }^{42} \mathrm{Ca}$ and other light magic nuclei offer abundant examples of relatively high $R_{4 / 2}$ values--even values near the collective regime of 2.0. (We will see later that the role of $R_{4 / 2}$ is nevertheless quite important, albeit for different kinds of information.) The yrast energies discussed above, however, may provide clues. Constant values suggest regions of good seniority since even small breaking of seniority can induce large effects.

It is important to note that the "seniority breaking" need not involve wave functions with components of different $v$ (e.g., $v=2,4, \ldots .$.$) . Variations in E\left(2_{1}^{+}\right)$and $\mathrm{E}\left(4_{1}^{+}\right)$can be produced with pure seniority $\mathrm{v}=2$ wave functions as long as the amplitudes are spread over several non-degenerate $\mathrm{j}$ orbits. This is illustrated in fig. 13 , which shows the energy difference of the $2_{1}^{+}$state for $n=6$ and $n=2$ particles, with $\mathrm{h}_{9 / 2}$ and $f_{7 / 2}$ orbits interacting under a $\delta$ function interaction, as a function of the single particle energy (s.p.e.) difference $\Delta \varepsilon \equiv \varepsilon_{7 / 2}-\varepsilon_{9 / 2}$. In these calculations we fixed the seniority as $v=0$ for the ground state and $v=2$ for the $2^{+}$level. Hence, any violation of the energy constancy required by good seniority cannot be due to seniority mixing but rather only to configuration mixing of non-degenerate orbits. For $\Delta \varepsilon=0$, the orbit degeneracy satisfies the conditions of generalized seniority and hence $\mathrm{E}\left(2_{1}^{+}\right)$is constant. For large $\Delta \varepsilon$, the interaction is not strong enough to excite particles to the $f_{7 / 2}$ orbit so that one has, effectively, a single $j$--good seniority situation. For intermediate $\Delta \varepsilon$ values the conditions for generalized seniority break down and $\mathrm{E}\left(2_{1}^{+}\right)$changes, although (see above), $\Delta \mathrm{E}_{4-2}$ may remain constant..

It is also possible to test the validity of magic numbers in new mass regions with $B(E 2)$ values. To do this, it is necessary to develop some global understanding of the variations of $B(E 2)$ values across the nuclear chart. Consider figs. 14 and 15 , which show ${ }^{10} B(E 2)$ values for all even-even nuclei as a function of $Z$ (fig. 14) and versus the valence nucleon product $N_{p} N_{n}$ in fig. 15. In fig. 14, while there are some evident trends, the data are still highly complex. They easily spread over an order- 
of-magnitude for a given $Z$ (and three orders-of-magnitude total--hence the need for a log scale). In contrast, in fig. 15a, the B(E2) values, which are expressed in W.u. and, additionally are normalized by another factor of $\mathrm{A}$, lie on a simple compact trajectory which is linear well into each shell. [A few specific nuclei that do not fit this tight correlation are omitted from fig. 15a. They are included in fig. $15 \mathrm{~b}$ and will be discussed later.] Of course, a prerequisite for fig. 15 is an accurate and known accounting of $\mathrm{N}_{\mathrm{p}} \mathrm{N}_{\mathrm{n}}$. If the shell structure is altered, from that defined by the traditional magic numbers, or is unknown, then the use of incorrect $N_{p}$ and $N_{n}$ values can generate large deviations from the empirical correlation. Two examples illustrate this. Figure 16 shows three plots of $B(E 2)$ values against $N_{p} N_{n}$ for the $A \sim 100$ region. On the left, the standard magic numbers are used, namely $Z=38,50$ for $50<N<60, Z=28,50$ for $N \geq 60$. A compact correlation is achieved. If the well known subshell at $\mathrm{Z}=38$ for $\mathrm{N}<60$ were not assumed, however, the correlation becomes messy. This is shown in the middle panel of fig. 16 which assumes proton magic numbers of $Z=28$ and 50 throughout.

We can use this approach to test other magic numbers. Suppose, for example, that $\mathrm{Z}=50$ is not taken as a magic number in constructing fig. 16. Then the plot on the right of fig. 16 is obtained. Clearly, the compact correlation for B(E2) values against $\mathrm{N}_{\mathrm{p}} \mathrm{N}_{\mathrm{n}}$ that is found in all other mass regions is destroyed. Even in advance of specific level scheme data on $100 \mathrm{Sn}$, this certainly suggests that the $\mathrm{Z}=50$ proton shell closure is intact, at least for nuclei within a few proton or neutron numbers of 50. Of course, it does not prove that ${ }^{100} \mathrm{Sn}$ itself is doubly magic since the entire precept we are discussing is that magic numbers can change, and it could still happen that the proton s.p.e.'s are different when $N=50$.

The second example concerns ${ }^{32} \mathrm{Mg}$. Although it has $\mathrm{N}=20$, it is a quasideformed nucleus. This suggests that $\mathrm{N}=20$ is not magic. We can test this notion using the $B(E 2)$ value. If $N=20$ were magic, then $N_{n}$ would be zero as would $N_{p} N_{n}$, 
giving a vanishing $B(E 2)$ value. If $N=20$ is not magic, then the nearest closed neutron shell is $N=28$, and $N_{p} N_{n}=4 \times 8=32$. One merely has to read the $B(E 2)$ value from fig. 15a. Correcting to $\mathrm{e}^{2} \mathrm{~b}^{2}$, one predicts $B\left(E 2: 2_{1}^{+} \rightarrow 0_{1}^{+}\right)=0.04 \mathrm{e}^{2} \mathrm{~b}^{2}$. This prediction is in agreement with the recently measured ${ }^{11}$ value of $0.046(9) e^{2} b^{2}$.

Although the following comment concerns collective nuclei rather than those near closed shells, a short digression is worthwhile since it highlights how global correlations can unveil otherwise inaccessible information and how simple data-energies and $B(E 2)$ values--can be much more informative than one might think.

The phenomenon of unit slope in plots (e.g., fig. 7) of $E\left(4_{1}^{+}\right)$vs. $E\left(2_{1}^{+}\right)$is actually part of a remarkable and nearly universal correlation. Extension of $\mathrm{E}\left(4_{1}^{+}\right)-\mathrm{E}\left(2_{1}^{+}\right)$plots to all types of nuclei, including collective species with $R_{4 / 2}>2.0$, reveals a new, tripartite, classification of nuclear structure from magic nuclei to deformed rotors. This is illustrated in fig. 17 for the $Z=50-82$ region. The data fall along a series of three straight line segments, with slopes 3.33, 2.00 and 1.0. The first of these is the rotor region: the last we have just discussed. The middle region is a surprising recent discovery ${ }^{12}$ that is not yet understood. All these collective, non-rotational nuclei $\left(2.05 \leq R_{4 / 2} \leq 3.15\right)$ have a slope of 2.00 and constant intercept. That is

$$
\mathrm{E}\left(4_{1}^{+}\right)=2 \mathrm{E}\left(2_{1}^{+}\right)+\varepsilon_{4}
$$

This is exactly the equation of an anharmonic vibrator (AHV) with constant anharmonicity $\varepsilon_{4}$. Note the widely varying $R_{4 / 2}$ values along the top scale. How such a wide variety of nuclei, with such different intrinsic or mean field structure, can exhibit almost constant anharmonicity is a central microscopic question raised by these results, as is the extremely rapid phase transition between rotor and AHV regions. 
Another critical question is whether approximately "global" (insofar as known nuclei are concerned) correlations such as the tripartite classification of structural evolution in fig. 17 are characteristic only of the nuclei already accessible near stability, and, if so, what is special about these nuclei, or, are they completely general and, if so, what generic features of the shell model and nucleonic interactions are involved. The answers to these questions go right to the heart of our basic understanding of nuclear structure.

The lower part of fig. 15 shows an unexpected use of the compact $B(E 2)-N_{p} N_{n}$ correlation. In contrast to fig. 15a, this plot includes a few points that deviate from the trendline. These deviations, of 20-30\%, would never have been visible in fig. 14 but clearly stand out in the global $\mathrm{N}_{\mathrm{p}} \mathrm{N}_{\mathrm{n}}$ plot. They do not correspond to random nuclei but to nuclei with large positive (points above the main trend) or negative (points below) hexadecapole deformations. An expansion of the quadrupole moment $[\propto B(E 2)]$ in multipole deformation components contains a term linear in $\beta_{4}$. Hence the $B(E)$ value, without the need for other, more difficult-to-obtain data, can even give clues to higher order deformations. It is worth stressing that the prerequisite for this is a compact, universal correlation.

Having looked at energies and $B(E 2)$ values separately, it is also informative to combine them. As we shall now see, a plot of $B(E 2)$ values against $R_{4 / 2}$ is even sensitive to the $\gamma$ degree of freedom 10,13 . Consider fig. 18 which plots two collective observables, $B(E 2)$ and $R_{4} / 2$, against each other. Individually, each can be a useful structural signature. Together they reveal even more information. For $R_{4 / 2} \geq 2.5$, the plot shows a 2-pronged trajectory. The structure of the nuclei along these two tracks is known. The upper track is primarily composed of nuclei in a spherical vibrator to rotor transitional region while the lower track consists of $\gamma$-soft $\rightarrow$ rotor nuclei. [The presence of some $\mathrm{A} \sim 130$ region $\mathrm{Ba}$ nuclei on the upper track is a bit 
puzzling and deserves further study of the relationship of $O(6)$ structure to structures enroute from $U(5)$ to $S U(3)$.]

Finally, there is another kind of signature, based on binding energies of neighboring even-even ground states, that can also signal either closed shell or phase transitional character. By forming double differences of binding energies, it is possible to isolate particular interactions amongst particular nucleons. There has been much study of the $\mathrm{p}-\mathrm{n}$ interaction, $\delta \mathrm{V}_{\mathrm{pn}}$, of the last proton and last neutron. ${ }^{14}$

More useful here is the non-pairing interaction of the last two neutrons (or protons, but we focus on neutrons to avoid the complication of the Coulomb interaction). Called $\delta \mathrm{V}_{\mathrm{nn}}$, it is defined ${ }^{15}$ in fig. 19: it is designed to remove the strong attractive pairing contribution. As is well known, what remains is actually a repulsive interaction $5,6,15$, as seen from empirical $\delta V_{n n}$ values in fig. 20 . The key points of the figure for our purposes are the spikes. Careful inspection of the defining formula for $\delta V_{n n}$ shows that it is invalid if the core for the $(N-1)^{\text {th }}, N^{\text {th }}$, and $(\mathrm{N}+1)^{\text {th }}$ neutrons is not the same. Hence $\delta \mathrm{V}_{\mathrm{nn}}$ is undefined if $\mathrm{N}$ is magic or if there is a phase transition. Nevertheless, numerically, $\delta V_{n n}$ values can still be obtained for such cases and are very informative. These "invalid" points are, in fact, the spikes in fig. 20, positive at magic numbers and negative at phase transitions. Thus, binding energies far off stability, which in some cases are easier and in other cases more difficult to obtain than excitation energies or $B(E 2)$ values, provide an alternate signature for shell structure or spherical-deformed transition regions.

\section{B. A Specific Example}

The work of Nazarewicz and colleagues 1,3 that we cited earlier on shell structure near the neutron drip lines is particularly intriguing. While more study is necessary, it is interesting to take their suggestion that the shell model potential itself is altered and that it lacks on $l^{2}$ term. Clearly, this will change the magic numbers in medium and heavy nuclei and also the traditional ordering of orbits 
within a shell. We illustrate this in fig. 21a, and we ask if there are simple empirical signatures of such effects. Figure 21 a shows the shell model orbits corresponding to 20-50 nucleons with a normal potential (the s.p.e.'s are taken from the Nilsson diagram at $\beta=0$ ) and, with the same potential with no $l^{2}$ term. With no $l^{2}$ term, the $f_{7 / 2}$ orbit rises in energy and so does the $g_{9 / 2}$ orbit. These "unique" parity orbits now are joined to others with the same parity. Hence $Z=50$ and 82 are no longer magic, being replaced by 40 and 70 . This effect could be disclosed by $B(E 2)-N_{p} N_{n}$ plots of very neutron-rich $Z=50$ nuclei. But there is an even more dramatic and immediate consequence. Recall the plot of $R_{4 / 2}$ vs. $A$ in fig. 9 and the discussion (fig. 11) of the correlation of $R_{4 / 2}$ values with $\Sigma a^{2}$. The principal point was that if $\Delta j=2$ orbital pairs are present, the $2^{+}$state is energetically favored, and hence $R_{4 / 2}$ increases. This is not the only effect at work (the binding energy of the $0_{1}^{+}$state, and hence the $2_{1}^{+}$and $4_{1}^{+}$excitation energies, also depends on the distribution of pairwise occupation of different $j$ orbits), but we focus on it here. In normal heavy nuclei, the unique parity orbit is lowered into the next lower shell, eliminating the principal $\Delta \mathbf{j}=2$ pair. Hence $R_{4 / 2} \sim 1.2-1.3$ for singly-magic medium and heavy-mass nuclei. Now, suppose the s.p.e.'s on the right in fig. 21a apply. Now a favored $g_{9 / 2}-d_{5 / 2}$ pair occurs low in the new shell. Hence one may expect $R_{4 / 2}$ to be anomalously large. This effect is illustrated in fig. 21b which shows calculations as a function of the $l^{2}$ strength. As anticipated, $R_{4 / 2}$ does indeed increase substantially (for a realistic $\delta$ function strength $V_{0}$ ). The lower panel of fig. 21 confirms that, as expected, the increase in $R_{4 / 2}$ as the $l^{2}$ term decreases is correlated with a rise in the occupation of $\Delta \mathrm{j}=2$ orbits by odd numbers of nucleons. The effect on $R_{4 / 2}$ is shown in fig. 22 which repeats fig. 12 but with a point corresponding to an " $l^{2}=0$ " calculation for 44 protons, that is, for 4 protons in the shell 40-70 that appears when the $l^{2}$ term vanishes. Observation of such anomalous $R$ values may therefore signal major changes in the shell model 
potential without the need for extensive transfer reaction data to establish s.p.e.'s and spectroscopic strengths in new regions.

This signature needs further study, especially with realistic shell-model calculations, to establish whether other effects could mask or swamp it. Such an effort, however, is certainly worthwhile: the idea that subtle details of the shell model potential can be revealed simply from the $\mathrm{E}\left(4_{1}^{+}\right) / \mathrm{E}\left(2_{1}^{+}\right)$ratio in a single magic nucleus is too appealing not to pursue.

\section{Conclusion}

We have discussed a number of signatures of structure that are remarkably simple, that are global, or nearly so, in character, that are based on simple ideas of the shell model and residual interactions, and, most importantly, which may be the easiest-to-obtain signatures of structure near closed shells in nuclei far from stability. Near the neutron drip line it seems plausible, ${ }^{1}$ perhaps even likely, that there may be radical departures from shell structure and nuclear structure compared to anything we have seen near stability. Hints of this already exist in the $\mathrm{N}=20$ region $\left({ }^{32} \mathrm{Mg}\right)$, in the $\mathrm{N}=\mathrm{Z}$ nucleus $80 \mathrm{Zr}$, and in the evanescence of shell structure in the $A=100$ ( $Z=38$ or 40 shell gap) and $A=150$ ( $Z=64$ gap) regions. The merging of bound levels into the continuum, the changes expected in the pairing interaction near zero binding energy, and the changes in the residual $p-n$ interaction when neutrons are filling much higher orbits than protons, will all contribute to engender new shell structure, even new shell model potentials, and new forms of collectivity.

With RNB's, we will need new and highly efficient signatures of structure. This paper has discussed a number of these, with emphasis on magic and nearmagic nuclei. Three of the most important are the energies, and energy differences, of the $2_{1}^{+}$and $4_{1}^{+}$states, their ratio $R_{4 / 2}$, and the $B\left(E 2: 2_{1}^{+} \rightarrow 0_{1}^{+}\right)$value.

The constancy of yrast energies can signal states of good seniority which, in turn, likely signals either an effectively single-j environment or a nearly-degenerate 
multi-j one. Non-constancy of these energies but constancy of their difference, can point to a pair addition mode where the $v \neq 0$ part of the wave functions of yrast states is nearly independent of valence nucleon number. The behavior of the $B(E 2)$ value for such nuclei can give evidence concerning the fractional filling of a shell if the nucleus is singly magic or, via an $\mathrm{N}_{\mathrm{p}} \mathrm{N}_{\mathrm{n}}$ analysis, concerning the validity of proposed magic numbers.

When near-closed shell, especially magic, nuclei are identified, the $R_{4 / 2}$ ratio can point to the underlying shell structure and even to the content of the shell model Hamiltonian itself (e.g., the presence or absence of an $l^{2}$ term). $\mathrm{B}\left(\mathrm{E} 2: 2_{1}^{+} \rightarrow 0_{1}^{+}\right.$) values for collective nuclei can also provide evidence for the $\gamma$ degree of freedom and for higher-order deformation multipoles (e.g., $\beta_{4}$ ). Finally, appropriate double differences of binding energies can also point both to magic nuclei (positive spikes in $\delta V_{n n}$ ) or to shape/phase transitions (negative spikes in $\delta V_{n n}$ ).

Nuclei far from stability will also allow us to test the remarkable nearlyuniversal correlations of structure that have recently been discussed, such as the tripartite classification of structure into rotor, AHV and pre-collective regimes via $E\left(4_{1}^{+}\right)-E\left(2_{1}^{+}\right)$plots, the smooth decrease of $R_{4 / 2}$ values for singly magic nuclei with $A$, the global $\mathrm{B}\left(\mathrm{E} 2: 2_{1}^{+} \rightarrow 0_{1}^{+}\right)$vs. $\mathrm{N}_{\mathrm{p}} \mathrm{N}_{\mathrm{n}}$ correlation, and the $\mathrm{B}\left(\mathrm{E} 2: 2_{1}^{+} \rightarrow 0_{1}^{+}\right)-\mathrm{R}_{4 / 2}$ two-tracked trajectory.

\section{Acknowledgements}

We would like to thank our collaborators in this and related work, in particular W.-T. Chou and D. S. Brenner, and are grateful to D. D. Warner, P. Federman, and A. Frank for discussions. Work supported by Contract No. DE-AC02-76CH00016 and DE-FG02- 88ER40417 with the United States Department of Energy. 


\section{References}

1. J. Dobaczewski et al., Phys. Rev. Lett. 72 (1994) 981.

2. P. Van Isacker, M. A. Nagarajan and D. D. Warner, Phys. Rev. C45 (1992) R13.

3. W. Nazarewicz, T. R. Werner and J. Dobaczewski, to be published.

4. M. M. Sharma et al., Phys. Rev. Lett. 72 (1994) 1431.

5. A. de Shalit and I. Talmi, Nuclear Shell Theory (Academic Press, New York, 1963).

6. R. F. Casten, Nuclear Structure from a Simple Perspective (Oxford University Press, New York, 1990).

7. N. V. Zamfir, R. F. Casten and D. S. Brenner, Phys. Rev. Lett. 72 (1994) 3480.

8. Y. K. Gambhir, S. Hag and J. K. Suri, Ann. Phys. (NY) 133 (1981) 154.

9. R. F. Casten, N. V. Zamfir and D. S. Brenner, Phys. Lett. B324 (1994) 267.

10. R. F. Casten and N. V. Zamfir, Phys. Rev. Lett. 70 (1993) 402.

11. T. Motobayashi et al., to be published.

12. R. F. Casten, N. V. Zamfir and D. S. Brenner, Phys. Rev. Lett. 71 (1993) 227.

13. N. V. Zamfir and R. F. Casten, Phys. Lett. B305 (1993) 317.

14. J.-Y. Zhang, R. F. Casten and D. S. Brenner, Phys. Lett. B227 (1989) 1.

15. N. V. Zamfir and R. F. Casten, Phys. Rev. C43 (1991) 2879. 
Table 1. Main components of the exact and pair addition mode $\left(h_{9 / 2} f_{7 / 2}\right)$ wave function probabilities (squared amplitudes) (in \%). From ref. 7.

\begin{tabular}{|c|c|c|c|c|c|c|c|c|}
\hline \multirow{3}{*}{$\mathrm{J}^{\pi}$} & \multicolumn{8}{|c|}{ number } \\
\hline & \multicolumn{2}{|r|}{2} & \multicolumn{3}{|c|}{4} & \multicolumn{3}{|c|}{6} \\
\hline & $w \cdot f^{a)}$ & exact $\left.{ }^{b}\right)$ & w.fa) & exact $\left.{ }^{b}\right)$ & pairc) & w.fa) & exact ${ }^{b)}$ & pairc) \\
\hline \multirow[t]{3}{*}{$0^{+}$} & $(20,00)$ & 74 & $(40,00)$ & 51 & 55 & $(40,20)$ & 51 & 45 \\
\hline & $(00,20)$ & 26 & $(20,20)$ & 43 & 38 & $(60,00)$ & 32 & 38 \\
\hline & & & $(00,40)$ & 6 & 7 & $(20,40)$ & 16 & 16 \\
\hline \multirow[t]{3}{*}{$2^{+}$} & $(22,00)$ & 92 & $(42,00)$ & 62 & 68 & $(42,20)$ & 41 & 36 \\
\hline & $(00,22)$ & 6 & $(22,20)$ & 27 & 24 & $(62,00)$ & 34 & 46 \\
\hline & $(11,11)$ & 2 & $(20,22)$ & 7 & 4 & $(40,22)$ & 9 & 5 \\
\hline \multirow[t]{3}{*}{$4^{+}$} & $(22,00)$ & 94 & $(42,00)$ & 64 & 70 & $(42,20)$ & 41 & 36 \\
\hline & $(11,11)$ & 4 & $(22,20)$ & 27 & 24 & $(62,00)$ & 35 & 47 \\
\hline & $(00,22)$ & 2 & $(31,11)$ & 5 & 3 & $(22,40)$ & 7 & 7 \\
\hline
\end{tabular}

a) This column lists the principal wave function components of the 2-orbit system, with the notation $\left[(n v)_{h_{9 / 2}},(n v)_{f_{7 / 2}}\right]$.

b) The "exact" wave function probabilities are those obtained by diagonalizing a surface $\delta$ interaction in the 2-orbit space with an interaction strength $V_{0}=1.0 \mathrm{MeV}$.

c) For $\mathrm{n}=4,6$, the "pair" column gives the "pair addition mode" wave function probabilities for $n$-particles in a state of spin J calculated by forming the product of the 2-particle $0^{+}$state (upper left box) with the (n-2)-particle wave function probabilities of spin J. 


\section{Figure Captions}

Fig. 1. Schematic depiction of proton and neutron levels near the neutron drip line.

Fig. 2. Levels of the configuration $\mid j^{2} J>$ with $j=7 / 2$ and a $\delta$ function interaction.

Fig. 3. Yrast energy levels of $\mathrm{v}=2$ states in a $\mathrm{j}^{\mathrm{n}}$ configuration. From ref. 6.

Fig. 4. Levels of the $\mathrm{Sn}$ isotopes.

Fig. 5. $B\left(E 2: 2_{1}^{+} \rightarrow 0_{1}^{+}\right)$values for a $j^{n}$ configuration with good seniority. The function plotted is $\mathrm{f}(1-\mathrm{f})$. See eq. 4 .

Fig. 6. $E\left(2_{1}^{+}\right), E\left(4_{1}^{+}\right)$and $\Delta E_{4-2}$ values. a) Magic proton nuclei, b) Magic neutron nuclei, c) Calculations (see text). Partly based on ref. 7.

Fig. 7. Plot of empirical $E\left(4_{1}^{+}\right)$against $E\left(2_{1}^{+}\right)$values for nuclei with $R_{4 / 2}<2.0$. The bottom panel shows calculations described in the text. From ref. 7.

Fig. 8. $B(E 2)$ values a) against mass number $A$ and $b$ ) against the fractional filling factor $f$ for singly magic nuclei with $Z, N \geq 50$. The curve on the right is the function $f(1-f)$ and is normalized to the data.

Fig. 9. Empirical $R_{4 / 2}$ values in singly magic nuclei.

Fig. 10. Schematic, semi-classical angular momentum diagram for the construction of a $2^{+}$state from angular momenta $j_{1}$ and $j_{2}$. Left: the co-planar case of $\left|j_{1} \pm j_{2}\right|=2$. Right: illustration of all other cases-- a $\Delta \mathrm{j}=1$ example. At the bottom, level schemes for these two cases are shown, for a surface $\delta$ function interaction with strength, $V_{0}$ $=1.0 \mathrm{MeV}$, and degenerate orbits. From ref. 9.

Fig. 11. Plots of $R_{4} / 2$ vs. $\Sigma a^{2}$, the summed percentage of wave function components corresponding to the occupation of each of two orbits, with $\Delta \mathrm{j}=2$, by an odd number of nucleons, for sets of shell model calculations with a surface $\delta$ interaction. Calculations for the 50-82 shell. The following calculations are included: s.p.e.'s as follows, g7/2 (0.0 MeV), d5/2 (0.8 MeV), $\mathrm{h}_{11 / 2}(1.9 \mathrm{MeV}), \mathrm{d}_{3 / 2}(3.5 \mathrm{MeV}), \mathrm{s}_{1 / 2}$ (3.9 $\mathrm{MeV}$ ), $\mathrm{V}_{0}=1.0 \mathrm{MeV}$, seniority $\mathrm{v} \leq 4$, up to 2 particles in the $h_{11 / 2}$ orbit, for 2, 4, 6, and 8 active particles (star symbols); same except for 4 particles and interaction 
strength, $\mathrm{V}_{0}$, varying from 0.2 to $1.2 \mathrm{MeV}$ (circles); 4 particles, $\mathrm{V}_{0}=1.0 \mathrm{MeV}$ and several choices for single particle energies (crosses).

Fig. 12. Shell model calculations of $R_{4 / 2}$ values for singly magic nuclei with a surface $\delta$ interaction. The calculations are done for 4 particles in each of several major shells. The solid line gives the results for a calculation with constant interaction strength, $\mathrm{V}_{\mathrm{o}}=0.8 \mathrm{MeV}$. The dashed lines give slightly more realistic results with an interaction strength that decreases with $A . V_{0}=1.2 \mathrm{MeV}$ for the 8-20 and $20-40$ shells, $0.6 \mathrm{MeV}$ for the $50-82$ shell and $0.3 \mathrm{MeV}$ for the $\mathrm{Z}>82$ shell.

Fig. 13. The energy difference $E\left(2_{1}^{+}\right)_{6}-E\left(2_{1}^{+}\right)_{2}$ for a system with $h_{9 / 2}$ and $f_{7 / 2}$ orbits as a function of the single particle energy difference $\Delta \varepsilon$ between the two orbits, for a $\delta$ function strength of $\mathrm{V}_{0}=1.0 \mathrm{MeV}$.

Fig. 14. $B(E 2)$ values for $Z \geq 40$. Based on ref. 10 .

Fig. 15. $B(E 2)$ values in W.u., divided by mass number $A$, plotted against $N_{p} N_{n}$. a) Excludes a few deviant points. This plot includes a bi-linear fit of the form $B(E 2)=$ $\mathrm{a}+\mathrm{b} \mathrm{N}_{\mathrm{p}} \mathrm{N}_{\mathrm{n}}$ where $\mathrm{a}=0.07, \mathrm{~b}=0.0065 \mathrm{~W} . \mathrm{u}$./A for $\mathrm{N}_{\mathrm{p}} \mathrm{N}_{\mathrm{n}}<175$, and $\mathrm{a}=1.14, \mathrm{~b}=0.0004$ W.u./A for $N_{p} N_{n}>175$. b) Data points for nuclei deviating from the main sequence are now included (filled circles). Based on ref. 10.

Fig. 16. $B(E 2)$ values for the $A=100$ region $(\mathrm{Zr}-\mathrm{Sn})$ against $\mathrm{N}_{\mathrm{p}} \mathrm{N}_{n}$ assuming normal shell and subshell closures (left), ignoring the $\mathrm{Z}=38$ subshell closure (middle), and ignoring in addition the $Z=50$ closure (right). Partly based on ref. 10.

Fig. 17. A plot, for the $Z=50-82, N=82-126$ region, of $E\left(4_{1}^{+}\right)$against $E\left(2{ }_{1}^{+}\right)$showing the division of the structural evolution into a tripartite classification of "seniority", anharmonic vibrator (AHV) and rotor (R) regions, corresponding to slopes of 1.0, 2.0, and 3.33. These regions are labelled at the bottom and demarcated by vertical dotted lines separated by narrow zones in which the structure varies rapidly in a way that resembles phase transitional behavior seen in other physical systems. From ref. 7. 
Fig. 18. Empirical $B(E 2)$ values against $R_{4 / 2}$ values for nuclei with $Z$ values from $50-$ 82. Similar to ref. 13.

Fig. 19. Definition of $\delta \mathrm{V}_{\mathrm{nn}}$ in terms of a double difference of binding energies. From ref. 15.

Fig. 20. Empirical $\delta V_{n n}$ for $N>30$. The solid lines connect isotopes. Based on ref. 15 . Fig. 21. a) Evolution of s.p.e.'s for nucleon numbers $20-50$ as a function of the strength of the $l^{2}$ term. b) Calculated $R_{4 / 2}$ values as a function of the strength of the $l^{2}$ term. c) Corresponding $\Sigma \mathrm{a}^{2}$ values.

Fig. 22. $R_{4 / 2}$ against $Z$. The dashed line is from fig 12 . The square symbol gives the result of a $\delta$-function calculation ( with $\mathrm{V}_{0}=0.6 \mathrm{MeV}$ ) for 4 particles in the 40-70 shell that results when there is no $l^{2}$ term in the shell model Hamiltonian. 
$\|+||||||||$

$1 \mid 1$

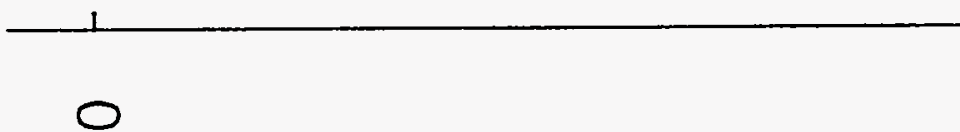

' ${ }^{\circ}{ }^{\circ}$ 


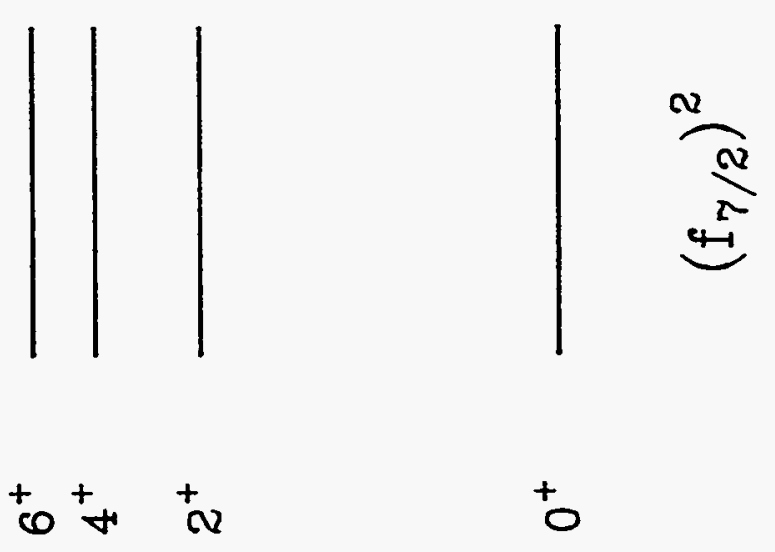



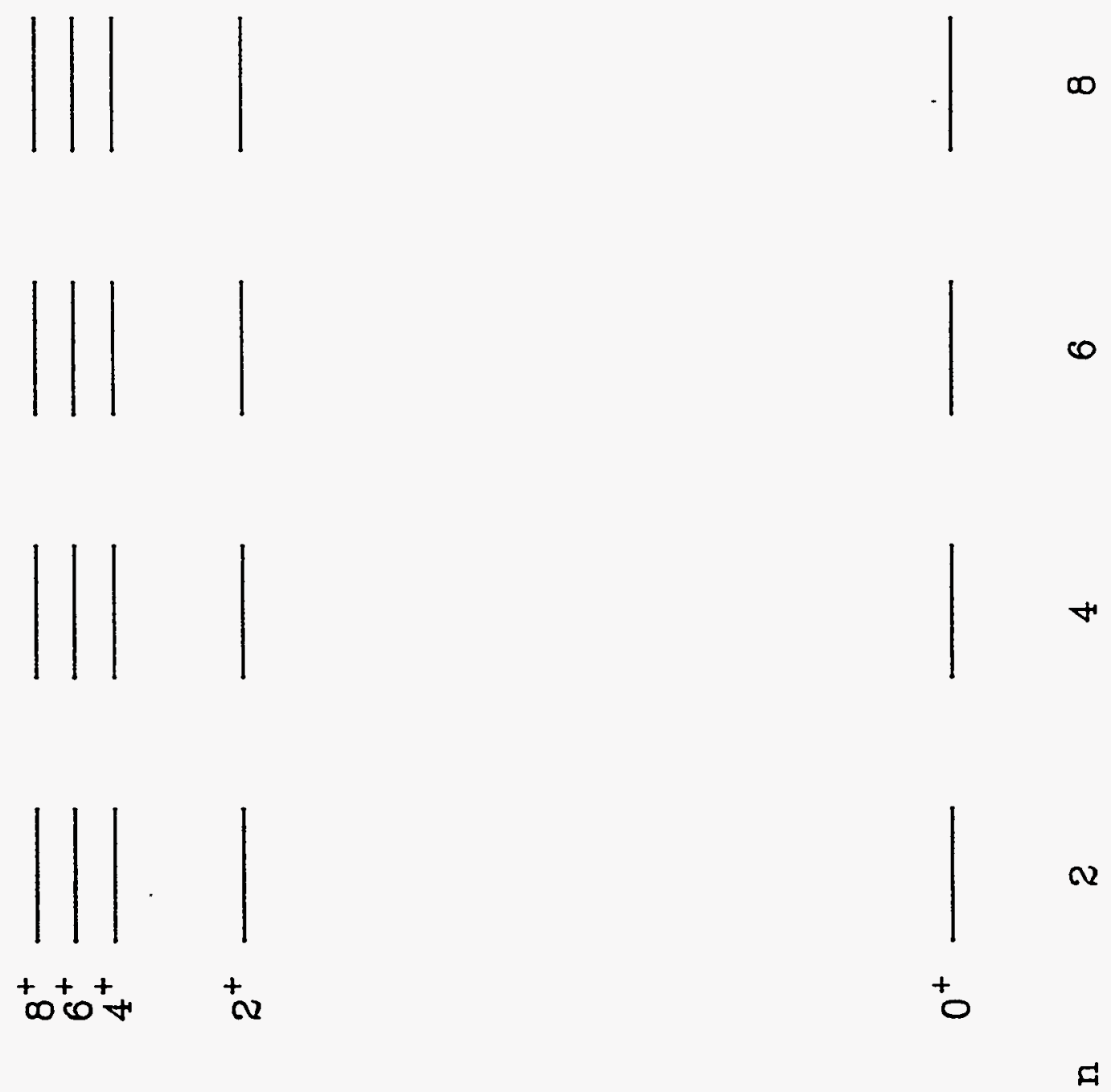

$>\quad$ Q

o 


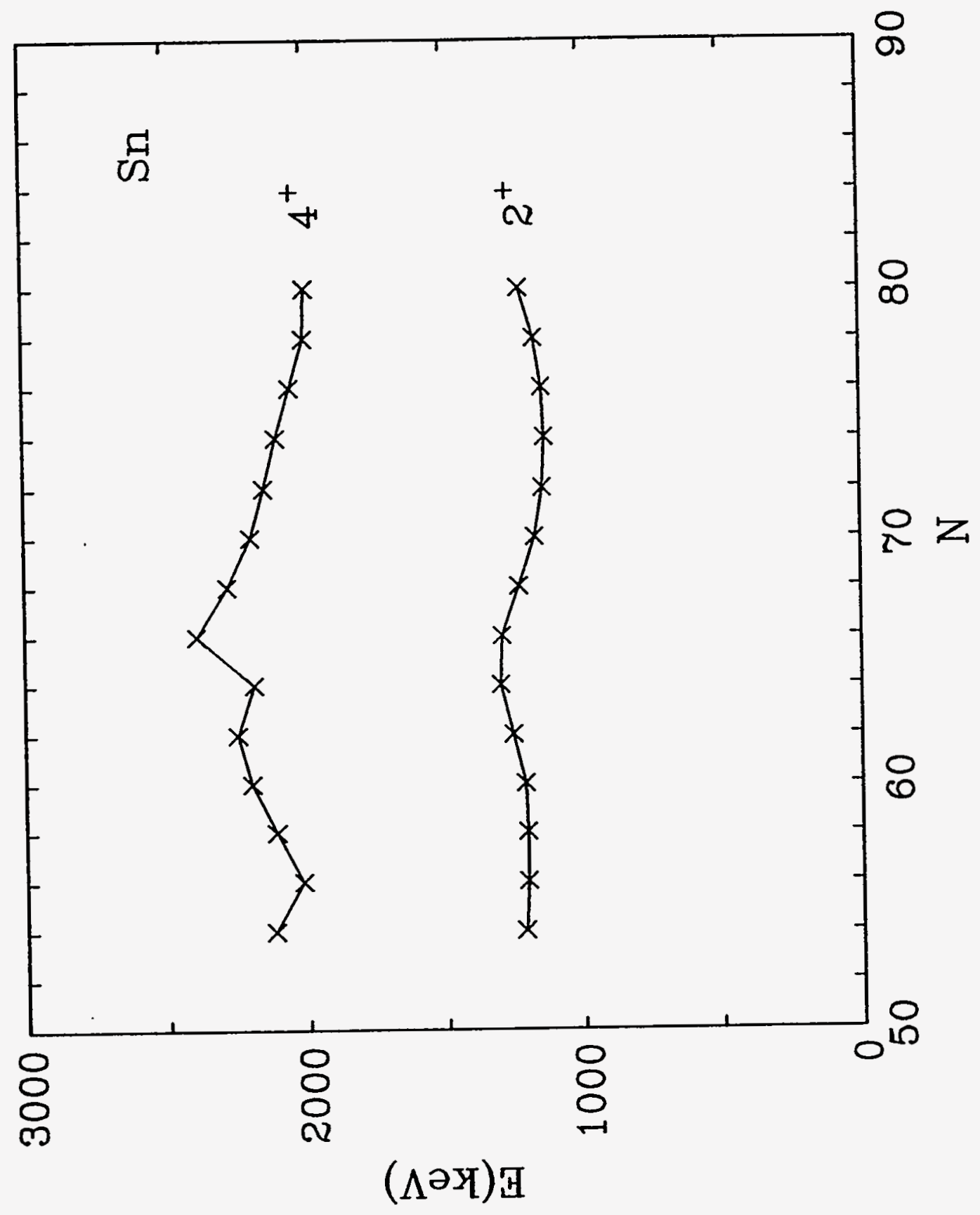




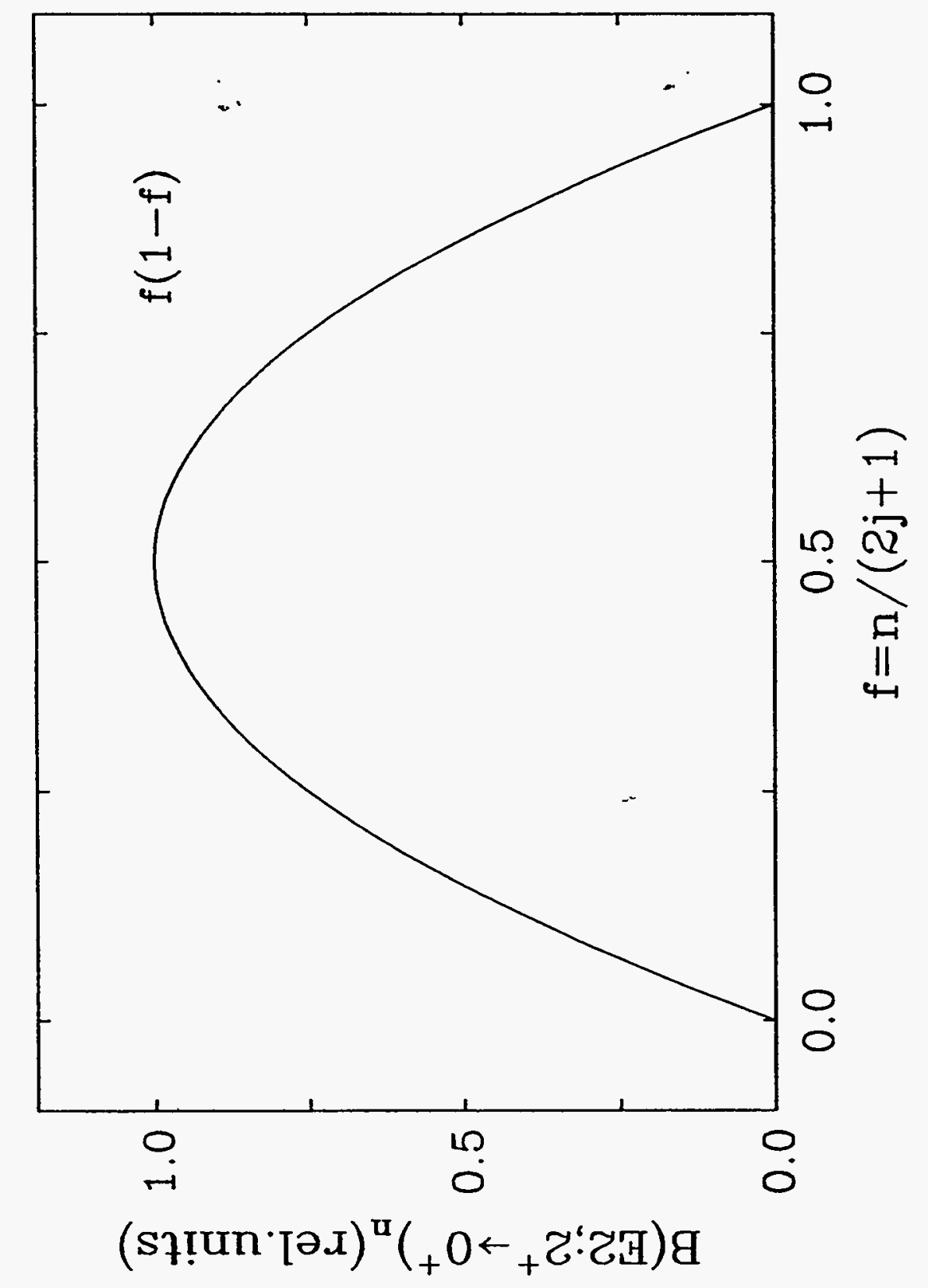




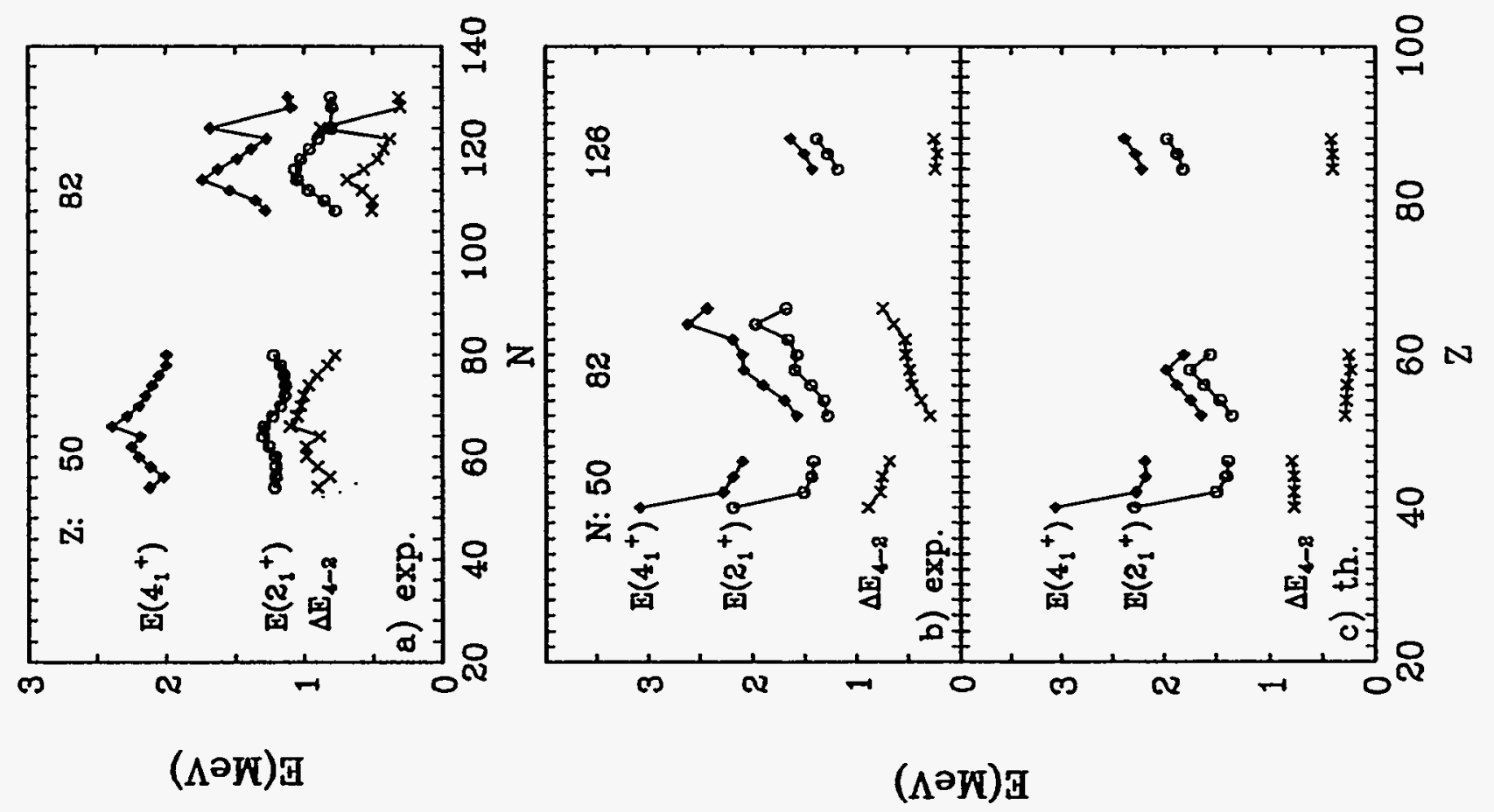




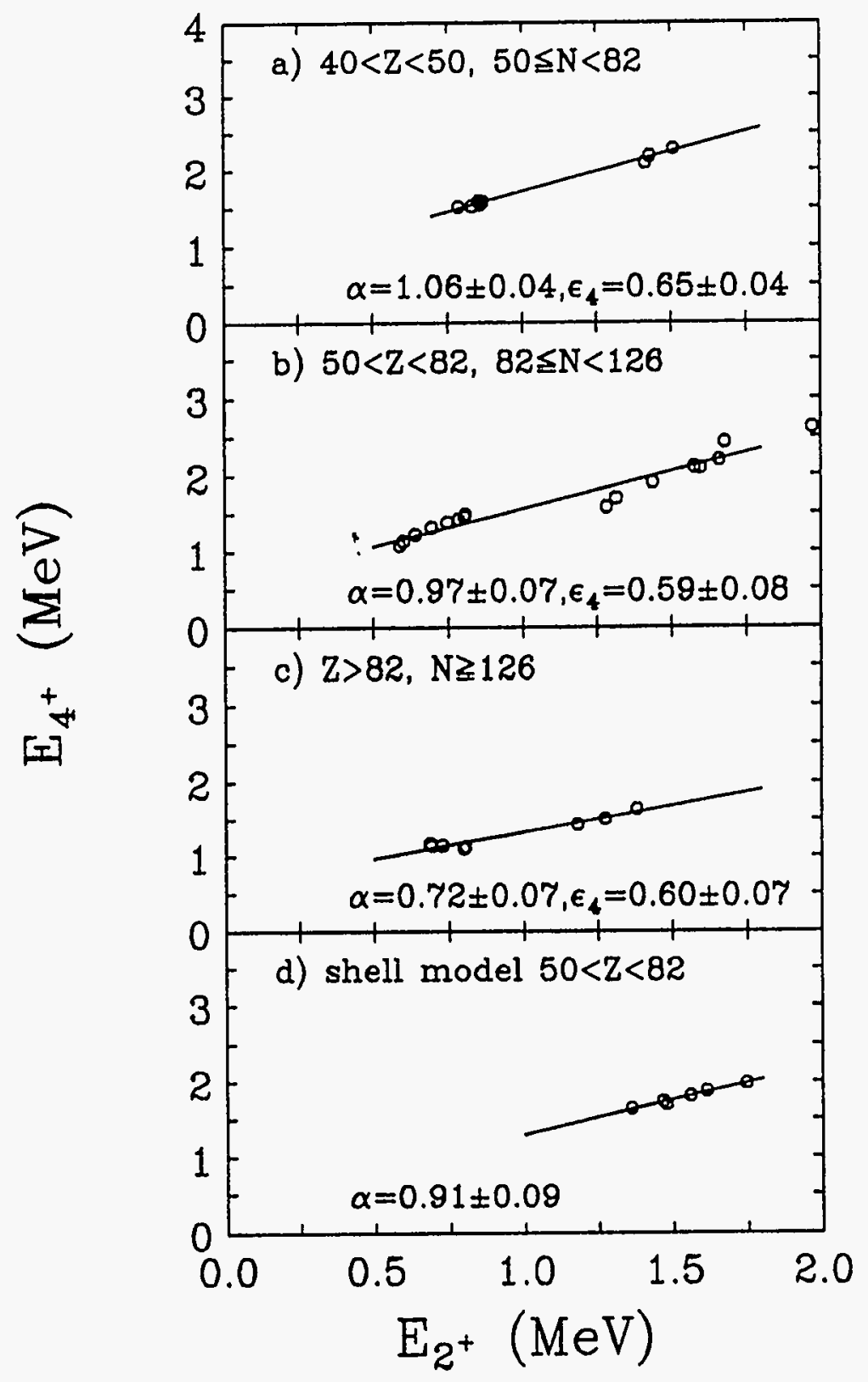




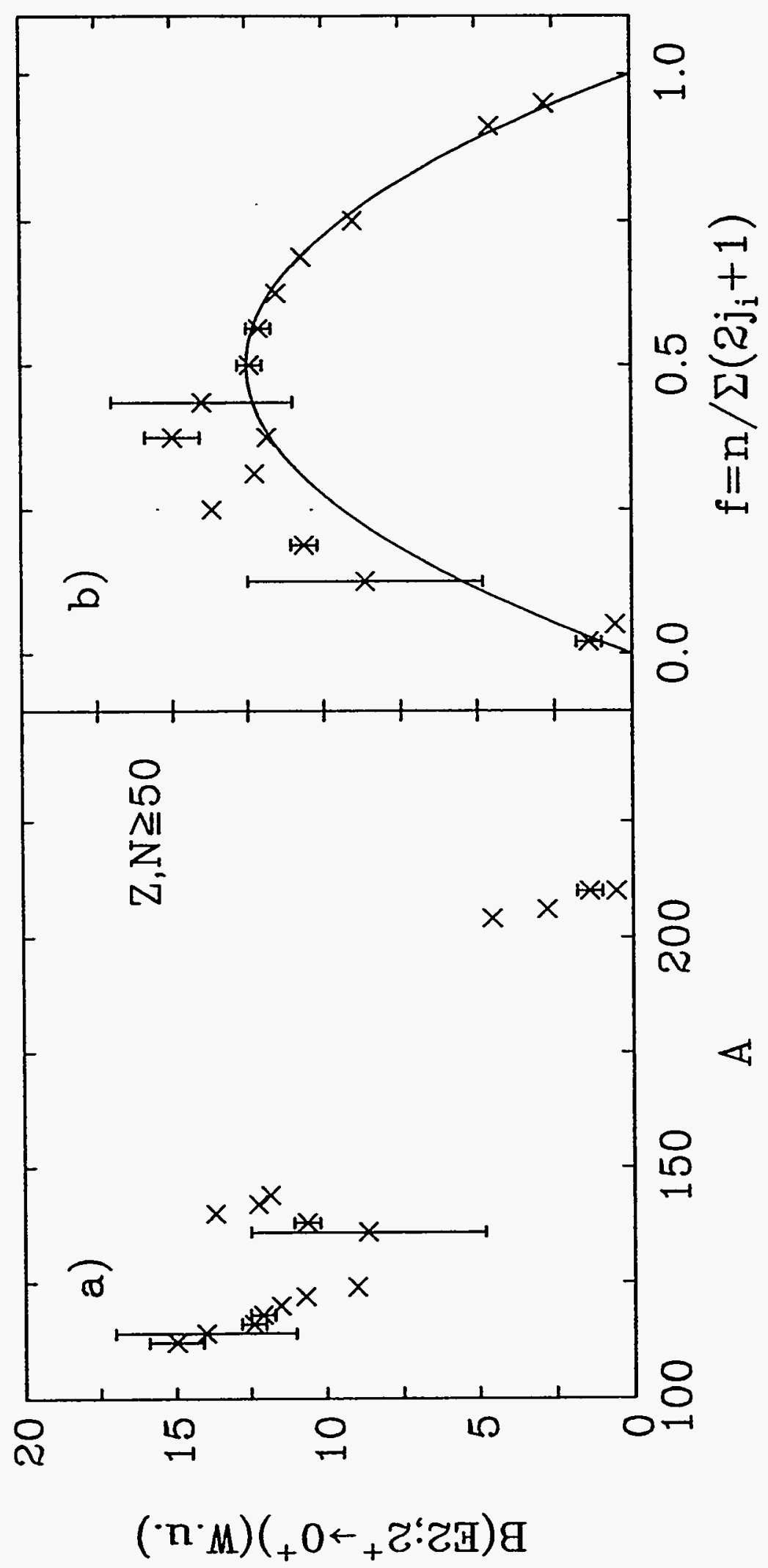




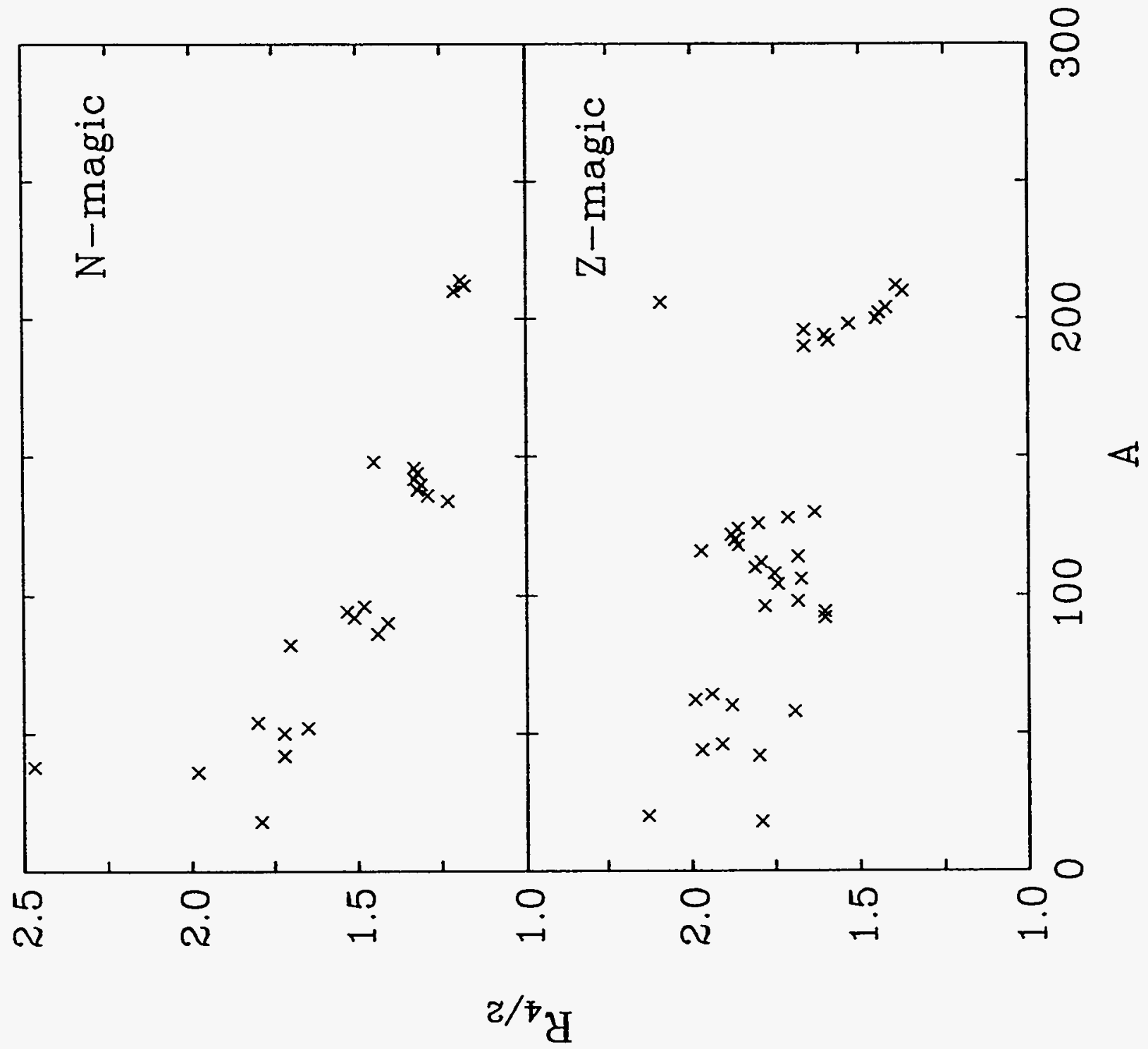




$$
\Delta \mathrm{j}=2
$$

$g_{7 / 2} d_{3 / 2}$
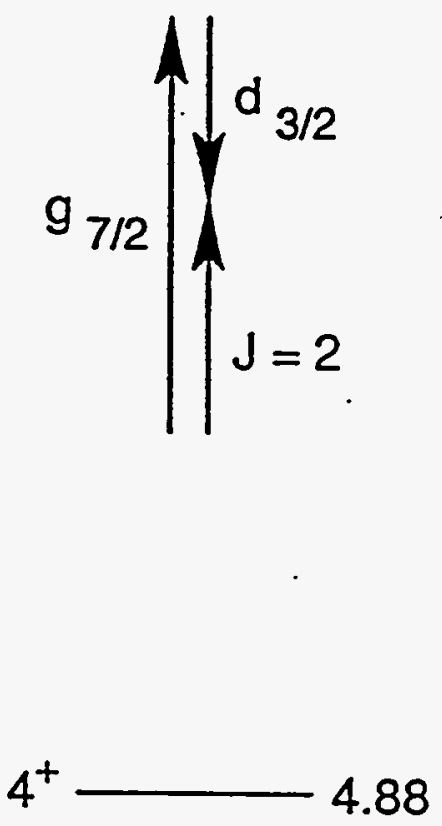

$\sum_{w}^{\frac{\delta}{W}} 2^{+}-2.62$

$$
\Delta \mathrm{j}=1
$$

$g_{7 / 2} d_{5 / 2}$
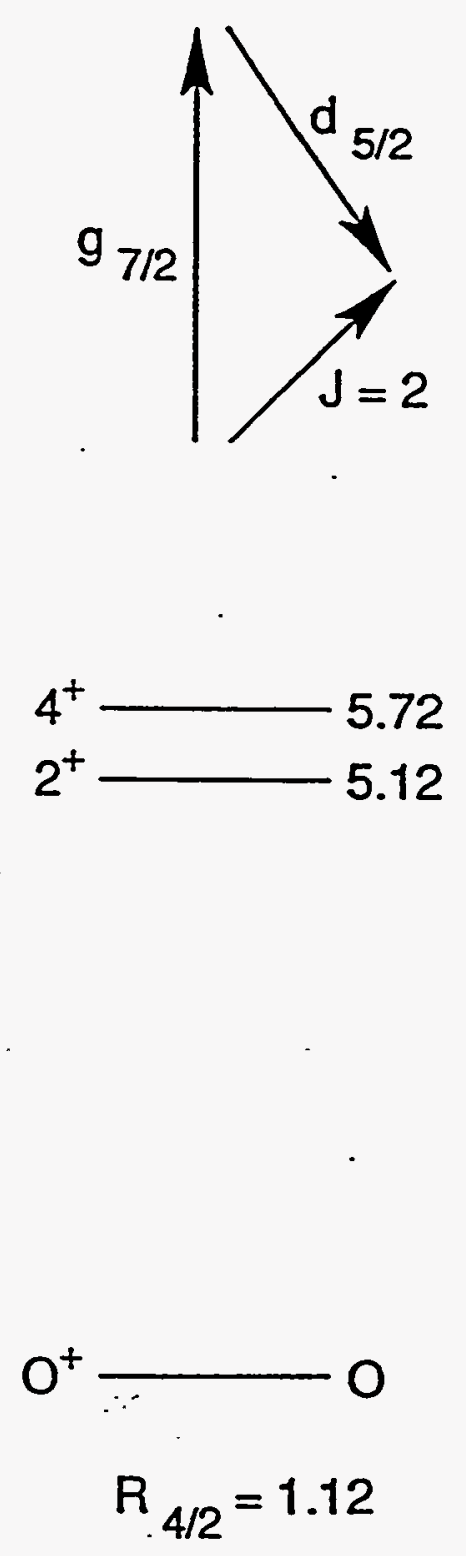


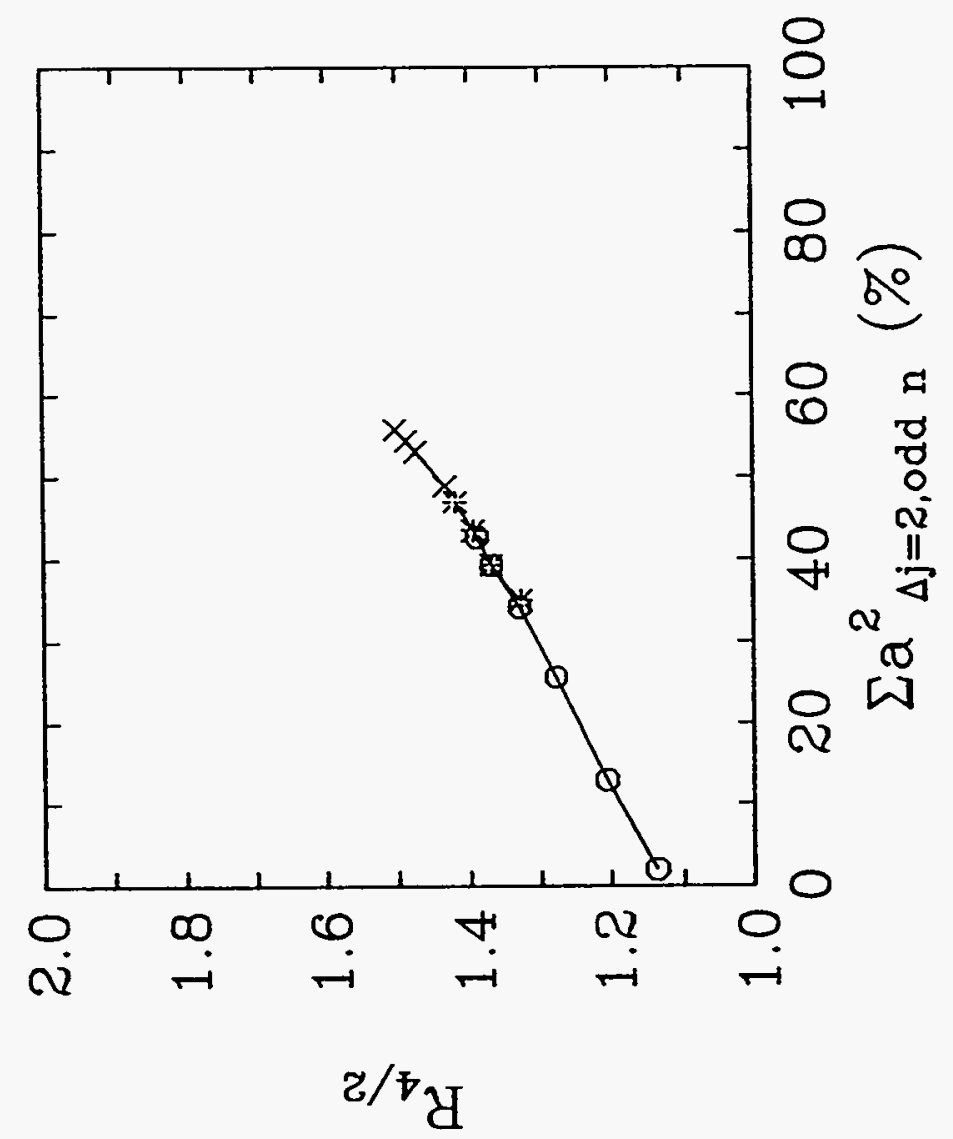




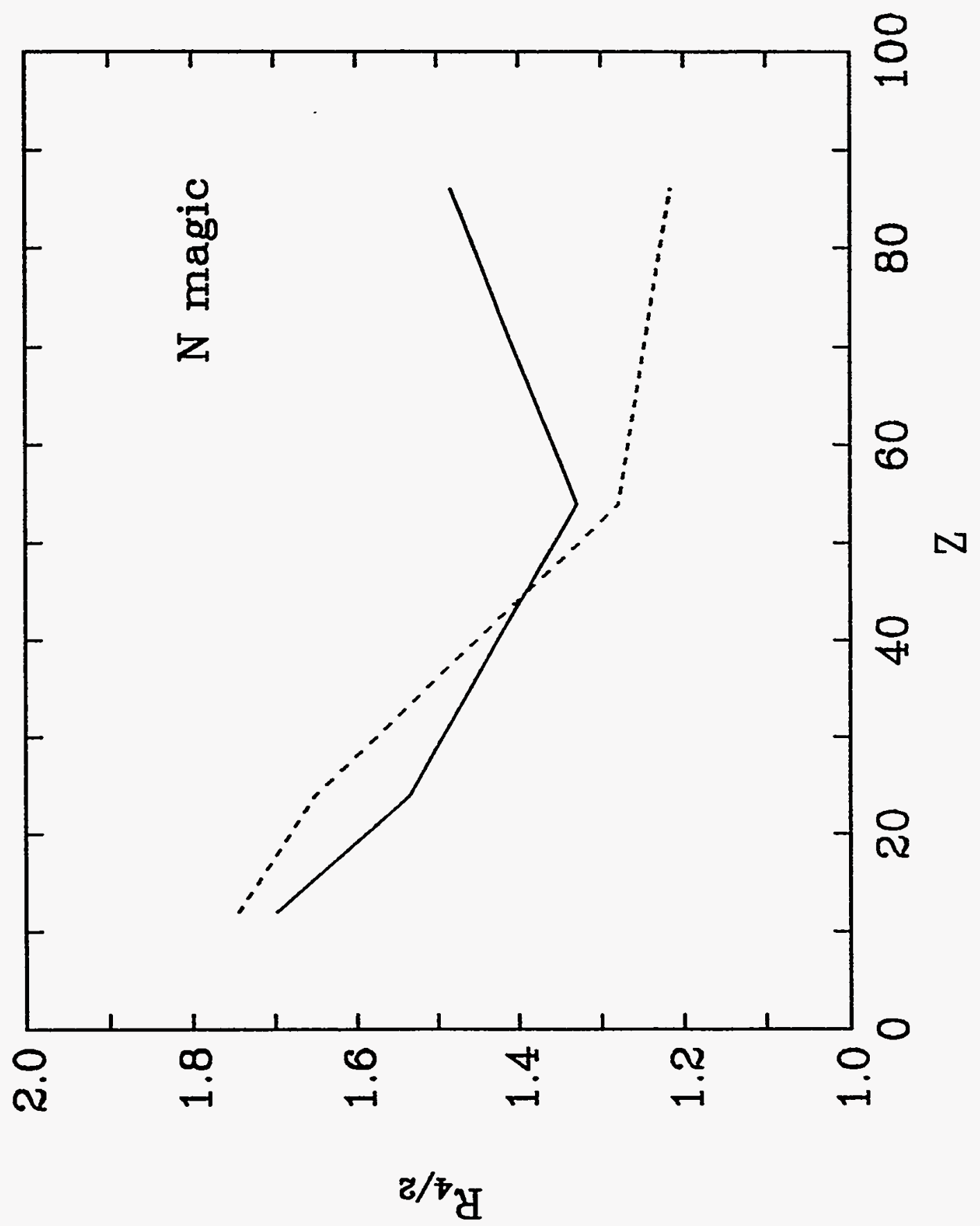




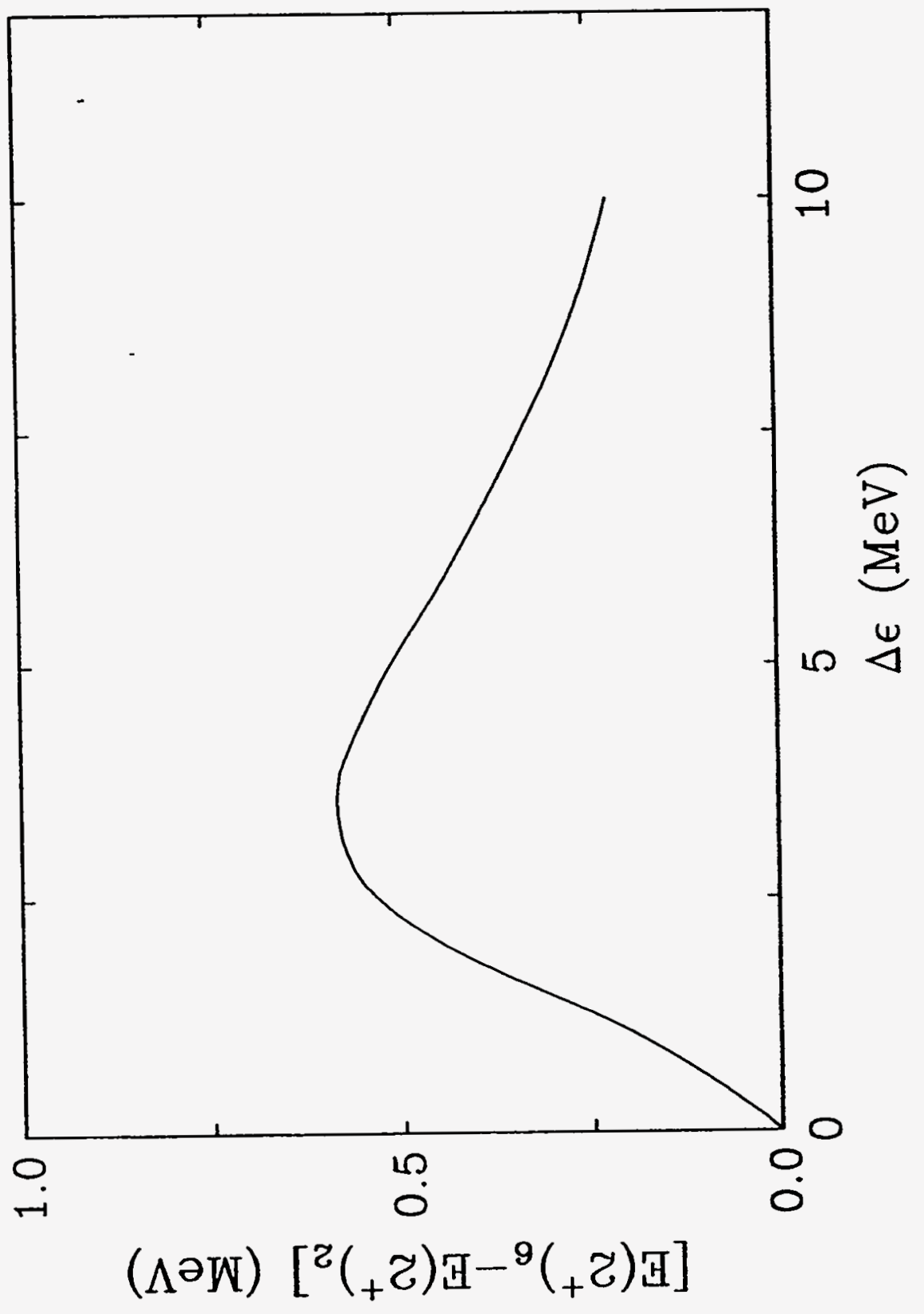




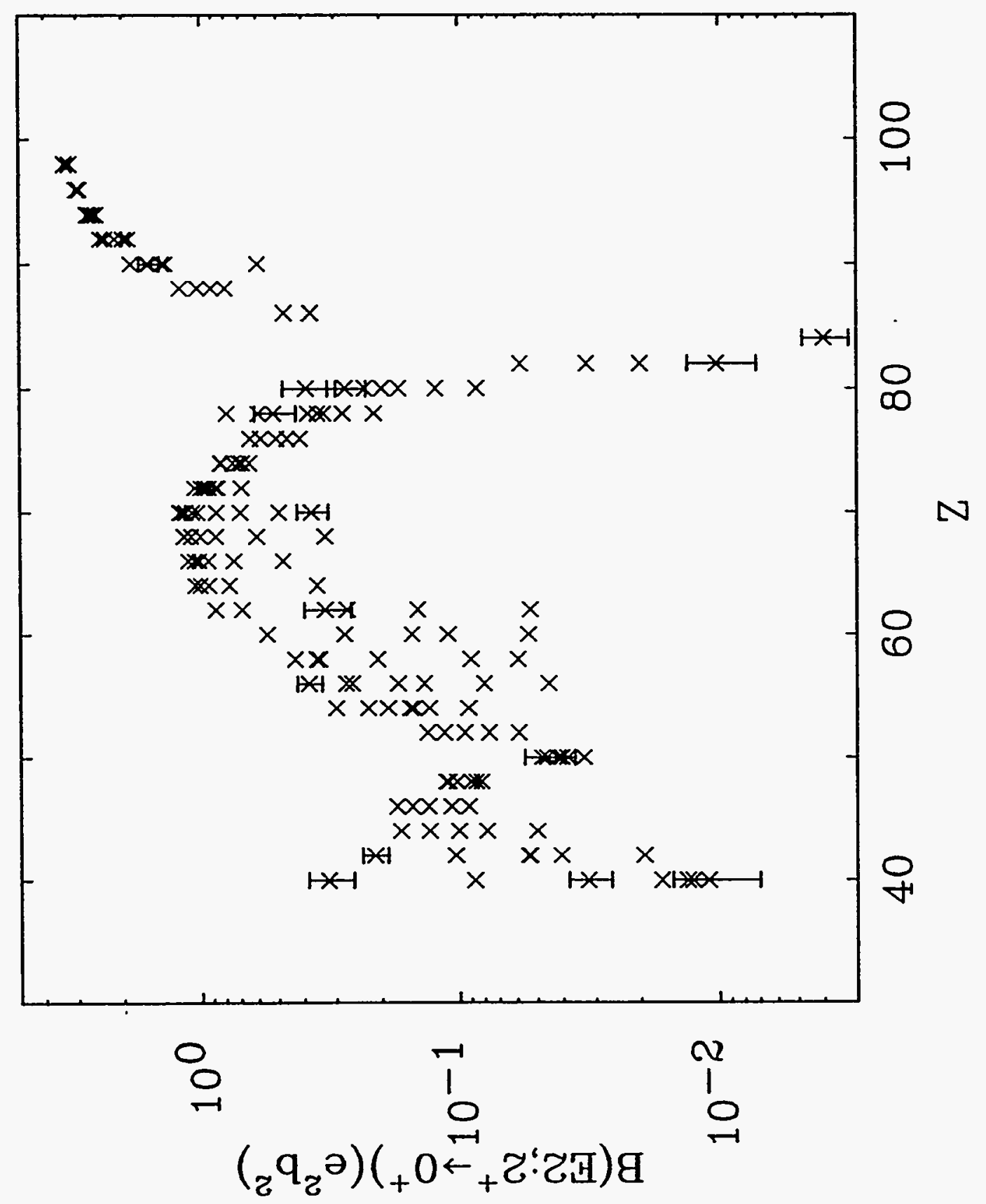




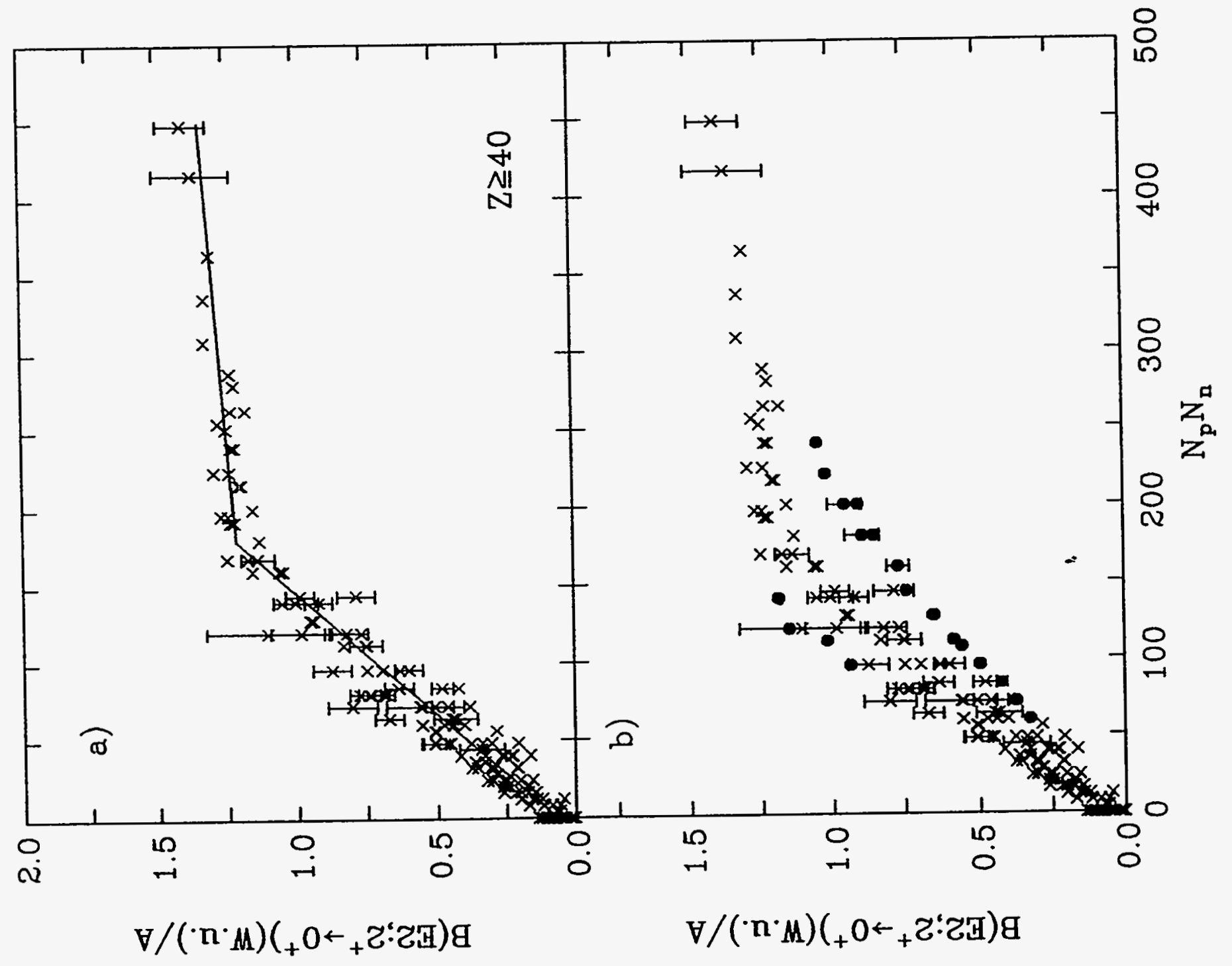




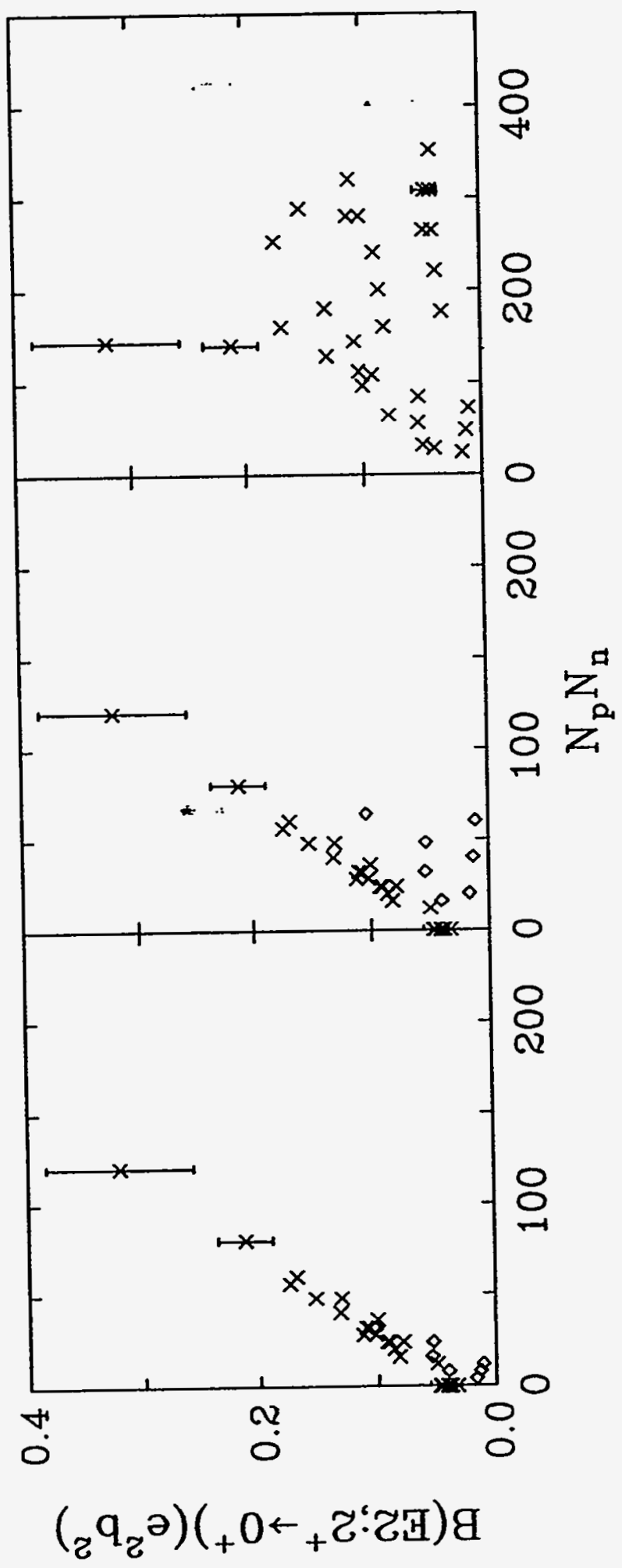




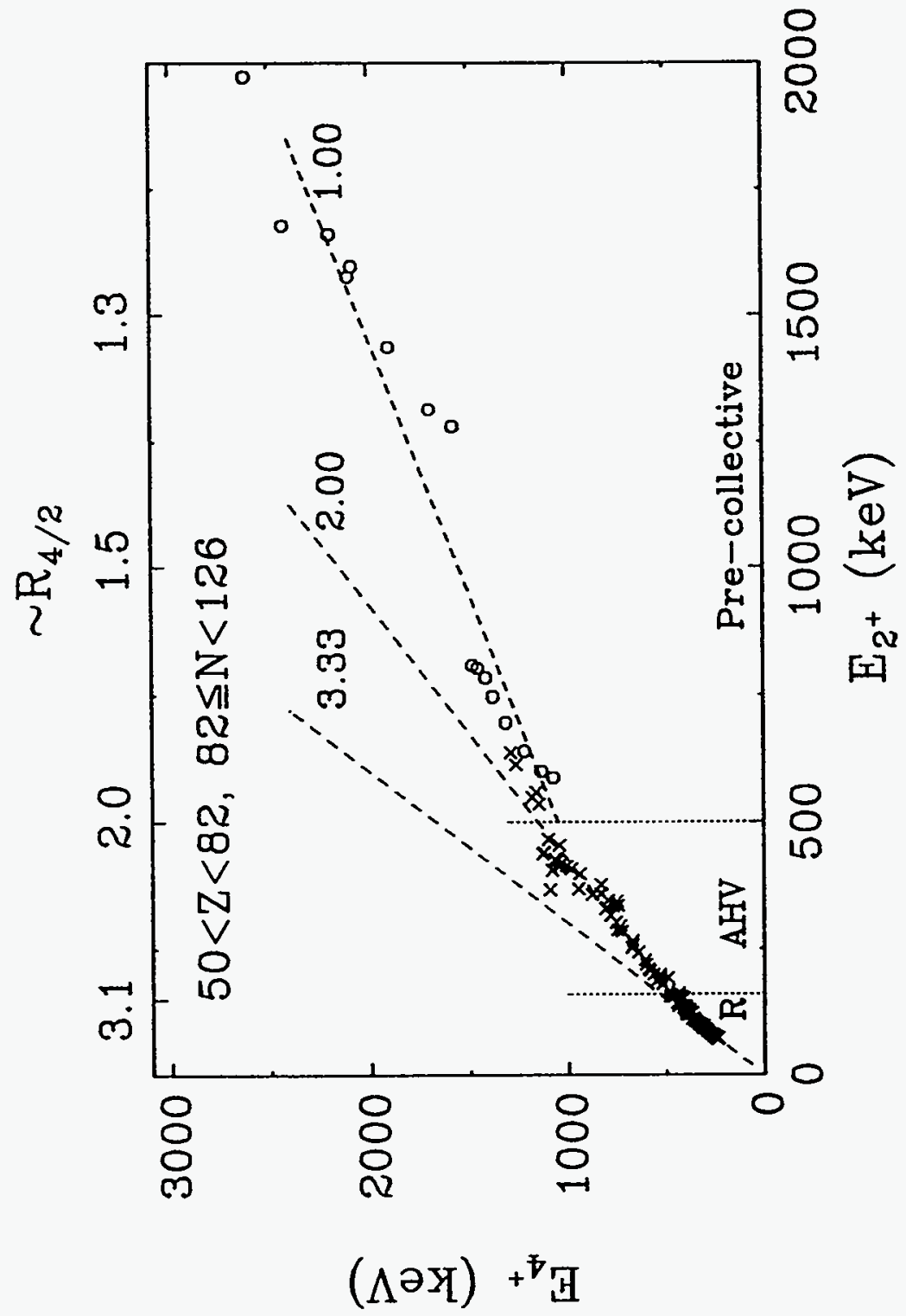




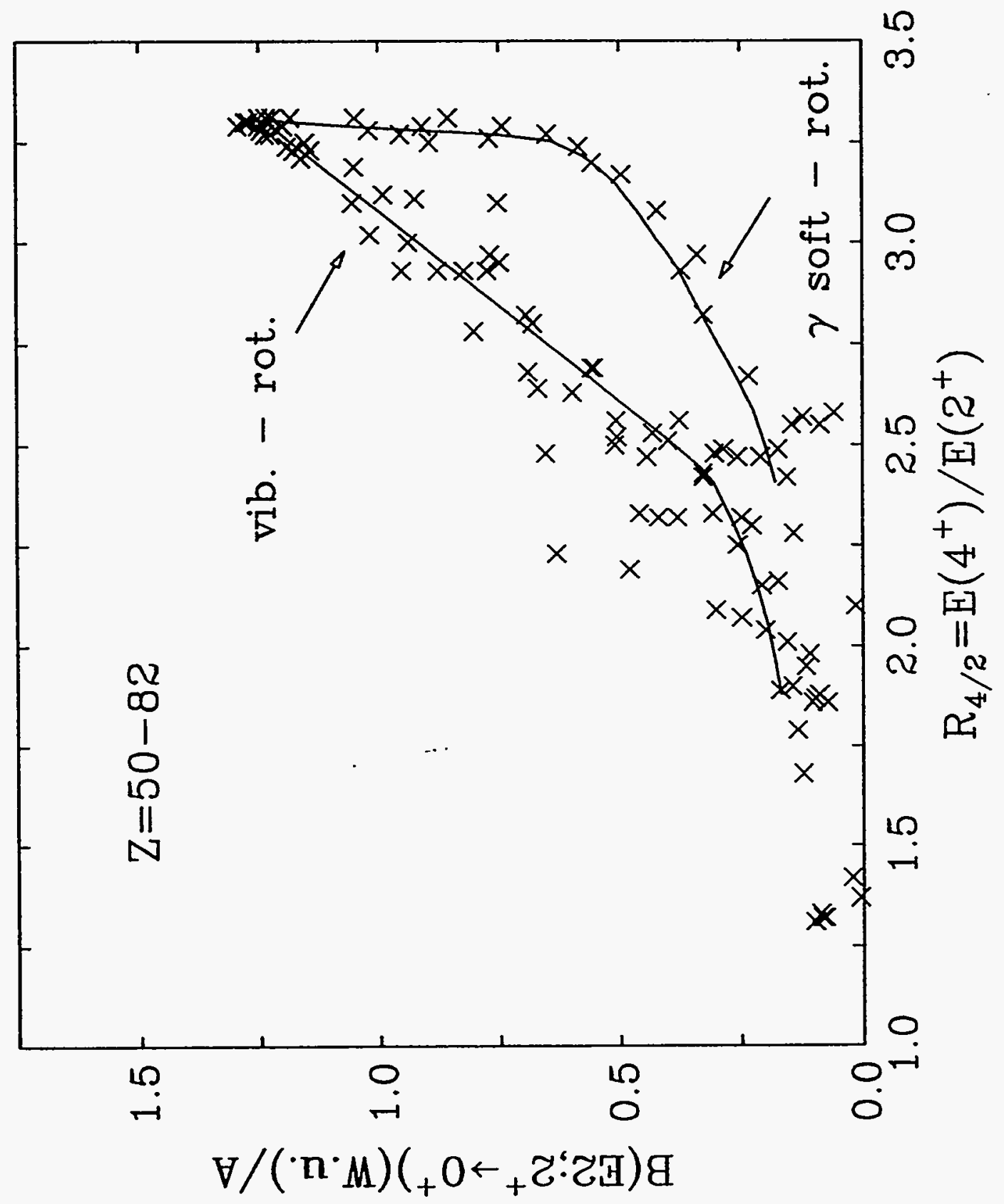




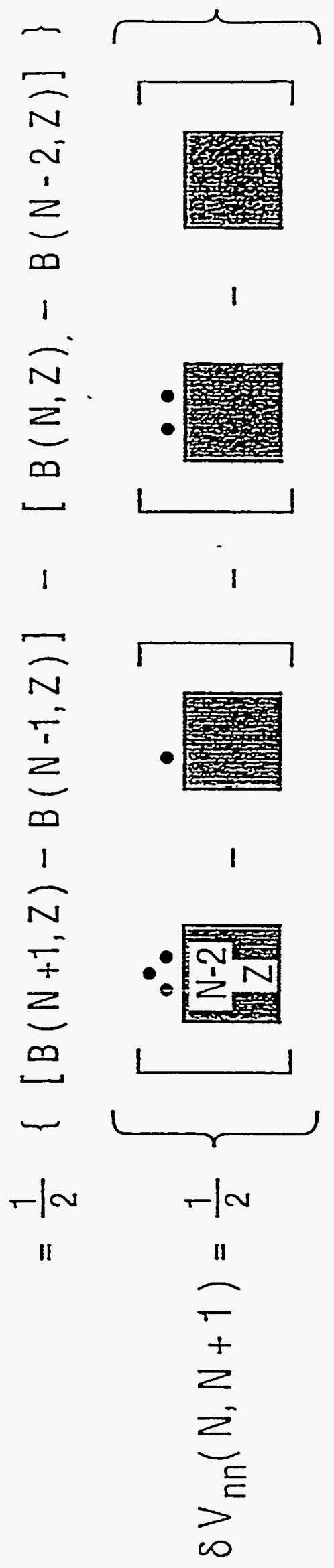




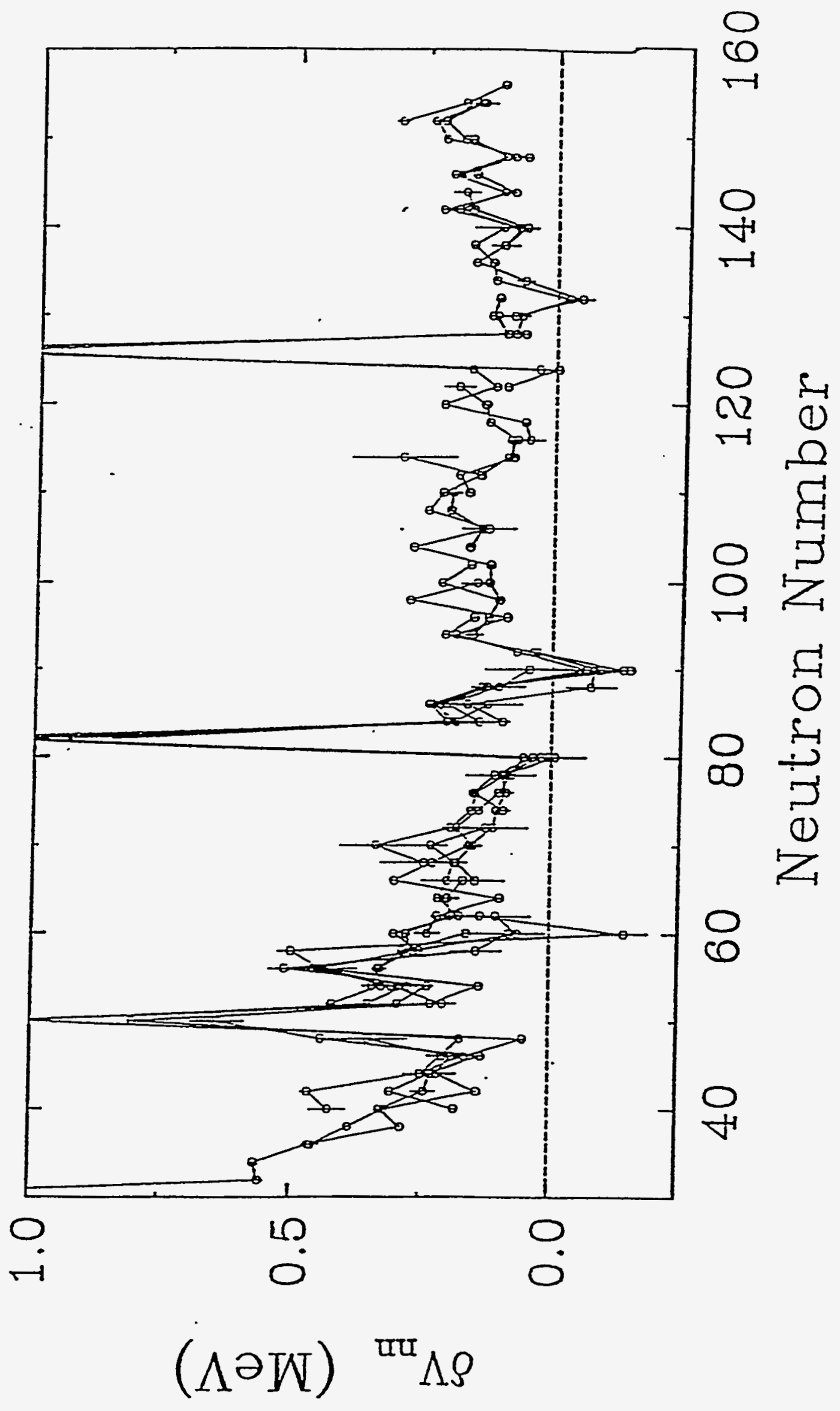



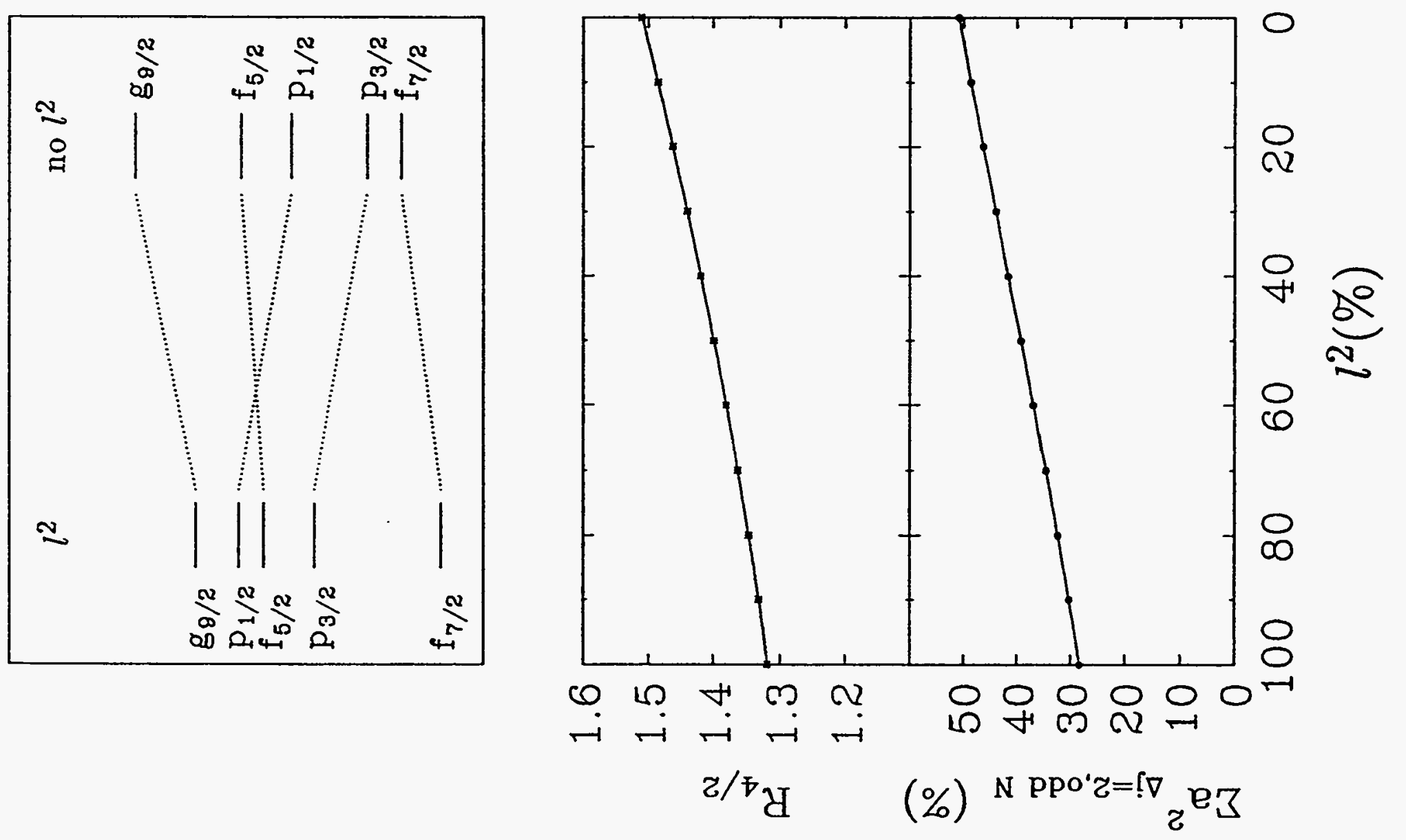


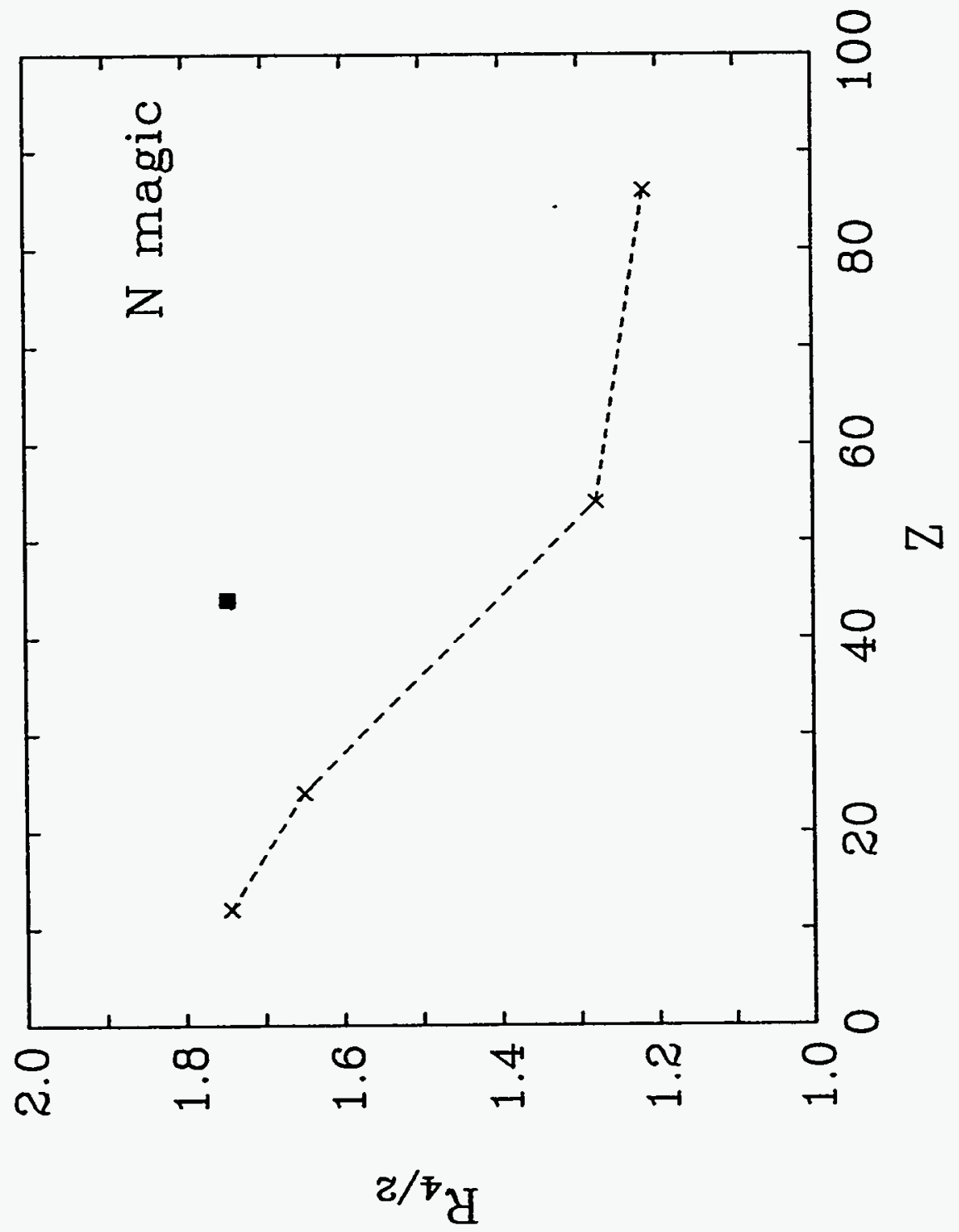

